\title{
Review
}

\section{Acute Mesenteric Ischemia in COVID-19 Patients}

\author{
Dragos Serban ${ }^{1,2, *,+} \mathbb{D}$, Laura Carina Tribus ${ }^{3,4,+}$, Geta Vancea $1,5,+\left(\mathbb{D}\right.$, Anca Pantea Stoian ${ }^{1}$ (D), \\ Ana Maria Dascalu ${ }^{1, *,+}{ }^{D}$, Andra Iulia Suceveanu ${ }^{6}$, Ciprian Tanasescu ${ }^{7,8}$, Andreea Cristina Costea ${ }^{9}$, \\ Mihail Silviu Tudosie ${ }^{1}$, Corneliu Tudor ${ }^{2}$, Gabriel Andrei Gangura ${ }^{1,10}$, Lucian Duta ${ }^{2}$ and \\ Daniel Ovidiu Costea ${ }^{6,11,+}$
}

Citation: Serban, D.; Tribus, L.C.; Vancea, G.; Stoian, A.P.; Dascalu, A.M.; Suceveanu, A.I.; Tanasescu, C.; Costea, A.C.; Tudosie, M.S.; Tudor, C.; et al. Acute Mesenteric Ischemia in COVID-19 Patients. J. Clin. Med. 2022, 11, 200. https://doi.org/10.3390/ jcm11010200

Academic Editor: Hiroki Tanabe

Received: 9 December 2021

Accepted: 27 December 2021

Published: 30 December 2021

Publisher's Note: MDPI stays neutral with regard to jurisdictional claims in published maps and institutional affiliations.

Copyright: (C) 2021 by the authors. Licensee MDPI, Basel, Switzerland. This article is an open access article distributed under the terms and conditions of the Creative Commons Attribution (CC BY) license (https:// creativecommons.org/licenses/by/ $4.0 /)$.
1 Faculty of Medicine, "Carol Davila" University of Medicine and Pharmacy, 020021 Bucharest, Romania; geta.vancea@umfcd.ro (G.V.); ancastoian@yahoo.com (A.P.S.); mihail.tudosie@umfcd.ro (M.S.T.); gabriel.gangura@umfcd.ro (G.A.G.)

2 Fourth Surgery Department, Emergency University Hospital Bucharest, 050098 Bucharest, Romania; lulutudor@gmail.com (C.T.); lucian.duta@gmail.com (L.D.)

3 Faculty of Dental Medicine, "Carol Davila" University of Medicine and Pharmacy, 020021 Bucharest, Romania; laura.tribus@umfcd.ro

4 Department of Internal Medicine, Ilfov Emergency Clinic Hospital Bucharest, 022104 Bucharest, Romania

“Victor Babes" Infectious and Tropical Disease Hospital Bucharest, 030303 Bucharest, Romania

6 Faculty of Medicine, Ovidius University Constanta, 900470 Constanta, Romania; andrasuceveanu@yahoo.com (A.I.S.); Daniel.costea@365.univ-ovidius.ro (D.O.C.)

7 Faculty of Medicine, Lucian Blaga University of Sibiu, 550024 Sibiu, Romania; ciprian.tanasescu@ulbsibiu.ro

8 Department of Surgery, Emergency County Hospital Sibiu, 550245 Sibiu, Romania

9 Department of Nephrology, Diaverum Clinic Constanta, 900612 Constanta, Romania; acostea2021@gmail.com

10 Second Surgery Department, Emergency University Hospital Bucharest, 050098 Bucharest, Romania

11 General Surgery Department, Emergency County Hospital Constanta, 900591 Constanta, Romania

* Correspondence: dragos.serban@umfcd.ro (D.S.); ana.dascalu@umfcd.ro (A.M.D.); Tel.: +40-72-330-0370 (D.S.); +40-72-740-2495 (A.M.D.)

+ These authors contributed equally to this work.

\begin{abstract}
Acute mesenteric ischemia is a rare but extremely severe complication of SARS-CoV-2 infection. The present review aims to document the clinical, laboratory, and imaging findings, management, and outcomes of acute intestinal ischemia in COVID-19 patients. A comprehensive search was performed on PubMed and Web of Science with the terms "COVID-19" and "bowel ischemia" OR "intestinal ischemia" OR "mesenteric ischemia" OR "mesenteric thrombosis". After duplication removal, a total of 36 articles were included, reporting data on a total of 89 patients, 63 being hospitalized at the moment of onset. Elevated D-dimers, leukocytosis, and $C$ reactive protein (CRP) were present in most reported cases, and a contrast-enhanced CT exam confirms the vascular thromboembolism and offers important information about the bowel viability. There are distinct features of bowel ischemia in non-hospitalized vs. hospitalized COVID-19 patients, suggesting different pathological pathways. In ICU patients, the most frequently affected was the large bowel alone $(56 \%)$ or in association with the small bowel (24\%), with microvascular thrombosis. Surgery was necessary in $95.4 \%$ of cases. In the non-hospitalized group, the small bowel was involved in $80 \%$, with splanchnic veins or arteries thromboembolism, and a favorable response to conservative anticoagulant therapy was reported in $38.4 \%$. Mortality was $54.4 \%$ in the hospitalized group and $21.7 \%$ in the non-hospitalized group $(p<0.0001)$. Age over 60 years $(p=0.043)$ and the need for surgery ( $p=0.019$ ) were associated with the worst outcome. Understanding the mechanisms involved and risk factors may help adjust the thromboprophylaxis and fluid management in COVID-19 patients.
\end{abstract}

Keywords: acute mesenteric ischemia; COVID-19; thromboemboembolism; SARS-CoV-2; endothelitis; cytokines; hypercoagulability 


\section{Introduction}

Acute mesenteric ischemia (AMI) is a major abdominal emergency, characterized by a sudden decrease in the blood flow to the small bowel, resulting in ischemic lesions of the intestinal loops, necrosis, and if left untreated, death by peritonitis and septic shock. In nonCOVID patients, the etiology may be mesenteric arterial embolism (in 50\%), mesenteric arterial thrombosis (15-25\%), venous thrombosis (5-15\%), or less frequent, from nonocclusive causes associated with low blood flow [1]. Several systemic conditions, such as arterial hypertension, atrial fibrillation, atherosclerosis, heart failure, or valve disease are risk factors for AMI. Portal vein thrombosis and mesenteric vein thrombosis can be seen with celiac disease [2], appendicitis [3], pancreatitis [4], and, in particular, liver cirrhosis and hepatocellular cancer [5].

Acute intestinal ischemia is a rare manifestation during COVID-19 disease, but a correct estimation of its incidence is challenging due to sporadic reports, differences in patients' selection among previously published studies, and also limitations in diagnosis related to the strict COVID-19 regulations for disease control and difficulties in performing imagistic investigations in the patients in intensive care units. COVID-19 is known to cause significant alteration of coagulation, causing thromboembolic acute events, of which the most documented were pulmonary embolism, acute myocardial infarction, and lower limb ischemia [6].

Gastrointestinal features in COVID-19 disease are relatively frequently reported, varying from less than $10 \%$ in early studies from China $[7,8]$ to $30-60 \%$, in other reports $[9,10]$. In an extensive study on 1992 hospitalized patients for COVID-19 pneumonia from 36 centers, Elmunzer et al. [7] found that the most frequent clinical signs reported were mild and self-limited in up to $74 \%$ of cases, consisting of diarrhea (34\%), nausea $(27 \%)$, vomiting $(16 \%)$, and abdominal pain (11\%). However, severe cases were also reported, requiring emergency surgery for acute bowel ischemia or perforation $[5,8]$.

The pathophysiology of the digestive features in COVID-19 patients involves both ischemic and non-ischemic mechanisms. ACE2 receptors are present at the level of the intestinal wall, and enterocytes may be directly infected by SARS-CoV-2. The virus was evidenced in feces and enteral walls in infected subjects [4,11-13]. In a study by $\mathrm{Xu}$ et al., rectal swabs were positive in 8 of 10 pediatric patients, even after the nasopharyngeal swabs became negative [14]. However, the significance of fecal elimination of viral ARN is still not fully understood in the transmission chain of the SARS-CoV-2 infection. On the other hand, disturbance of lung-gut axis, prolonged hospitalization in ICU, and the pro coagulation state induced by SARS-CoV-2 endothelial damage was incriminated for bowel ischemia, resulting in intestinal necrosis and perforation [8,9,15]. Early recognition and treatment of gastrointestinal ischemia are extremely important, but it is often challenging in hospitalized COVID-19 patients with severe illness.

The present review aims to document the risk factors, clinical, imagistic, and laboratory findings, management, and outcomes of acute intestinal ischemic complications in COVID-19 patients.

\section{Materials and Methods}

A comprehensive search was performed on PubMed and Web of Science with the terms "COVID-19" AND ("bowel ischemia" OR "intestinal ischemia" OR "mesenteric ischemia" OR "mesenteric thrombosis"). All original papers and case reports, in the English language, for which full text could be obtained, published until November 2021, were included in the review. Meeting abstracts, commentaries, and book chapters were excluded. A hand search was performed in the references of the relevant reviews on the topic.

\subsection{Data Extraction and Analysis}

The review is not registered in PROSPERO. A PRISMA flowchart was employed to screen papers for eligibility (Figure 1) and a PRISMA checklist is presented as a Supple- 
mentary File S1. A data extraction sheet was independently completed by two researchers, with strict adherence to PRISMA guidelines.

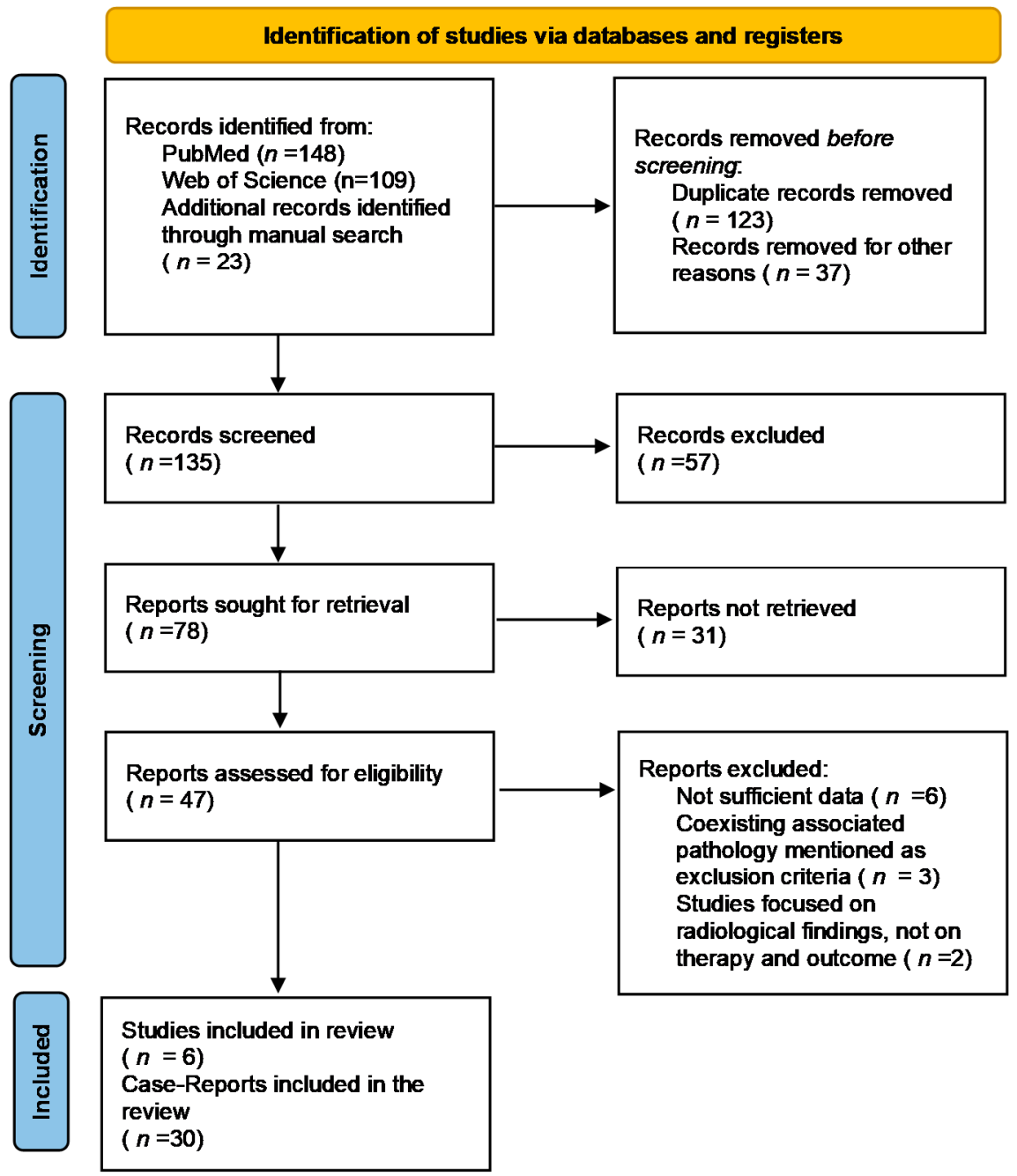

Figure 1. PRISMA 2020 flowchart for the studies included in the review.

The relevant data abstracted from these studies are presented in Tables 1-3. COVID-19 diagnosis was made by PCR assay in all cases. All patients reported with COVID-19 disease and mesenteric ischemia were documented in terms of age, sex, comorbidities, time from SARS-CoV-2 infection diagnosis, presentation, investigations, treatment, and outcome. A statistical analysis of the differences between acute intestinal ischemia in previously nonhospitalized vs. previously hospitalized patients was performed. The potential risk factors for an adverse vital prognosis were analyzed using SciStat ${ }^{\circledR}$ software (www.scistat.com (accessed on 25 November 2021)).

Papers that did not provide sufficient data regarding evaluation at admission, documentation of SARS-CoV-2 infection, or treatment were excluded. Patients suffering from other conditions that could potentially complicate intestinal ischemia, such as liver cirrhosis, hepatocellular carcinoma, intraabdominal infection (appendicitis, diverticulitis), pancreatitis, and celiac disease were excluded. Any disagreement was solved by discussion. 
Table 1. Patients with intestinal ischemia in retrospective studies on hospitalized COVID-19 patients.

\begin{tabular}{|c|c|c|c|c|c|c|c|c|c|}
\hline Study & $\begin{array}{l}\text { No of Patients with } \\
\text { Gastrointestinal } \\
\text { Ischemia (Total No of } \\
\text { COVID-19 Patients in } \\
\text { ICU) }\end{array}$ & $\operatorname{Sex}(M ; F)$ & $\begin{array}{c}\text { Age } \\
\text { (Mean) }\end{array}$ & BMI & $\begin{array}{l}\text { Time from } \\
\text { Admission to Onset } \\
\text { (Days) }\end{array}$ & Abdominal CT Signs & $\begin{array}{l}\text { Intraoperative/Endoscopic } \\
\text { Findings }\end{array}$ & Treatment & Outcomes \\
\hline Kaafarani HMA [16] & $5(141) ; 3.8 \%$ & $1 ; 3$ & 62.5 & 32.1 & 51.5 (18-104) days & NA & $\begin{array}{c}\text { Cecum-1-patchy necrosis } \\
\text { Cecum_ileon-1 } \\
\text { Small bowel-3; yellow discoloration on } \\
\text { the antimesenteric side of the small } \\
\text { bowel; } 1 \text { case + liver necrosis }\end{array}$ & Surgical resection & NA \\
\hline Kraft M [17] & $\begin{array}{l}4(190) ; \\
2.1 \%\end{array}$ & NA & NA & NA & NA & NA & $\begin{array}{l}\text { Bowel ischemia + perforation (2) } \\
\text { Bowel ischemia + perforation (1) } \\
\text { MAT+massive bowel ischemia (1) }\end{array}$ & $\begin{array}{l}\text { Right hemicolectomy (2) } \\
\text { Transverse colectomy (1) } \\
\text { Conservative, not fit for } \\
\text { surgery }\end{array}$ & $\begin{array}{l}\text { Recovery (3) } \\
\text { Death (1) }\end{array}$ \\
\hline Yang C [18] & $\begin{array}{l}20 \text { (190 in ICU; } 582 \text { in } \\
\text { total); } 10.5 \%\end{array}$ & $15: 5$ & 69 & 31.2 & $26.5(17-42)$ & $\begin{array}{c}\text { Distension } \\
\text { Wall thickness } \\
\text { Pneumatosis intestinalis } \\
\text { Perforation } \\
\text { SMA or celiac thrombosis }\end{array}$ & no info & $\begin{array}{c}\text { Right hemicolectomy } \\
7(35 \%) \\
\text { Sub/total colectomy12 } \\
(60 \%) \\
\text { Ileocecal resection } 1(5 \%)\end{array}$ & $\begin{array}{l}\text { Recovery (11) } \\
\text { Death (9) }\end{array}$ \\
\hline Hwabejire J [19] & 20 & $13: 7$ & 58.7 & 32.5 & $13(1-31)$ & $\begin{array}{c}\text { Pneumatosis intestinalis } 42 \% \\
\text { Portal venous gas ( } 33 \%) \\
\text { Mesenteric vessel patency } \\
92 \%\end{array}$ & $\begin{array}{c}\text { large bowel ischemia (8) } \\
\text { small bowel ischemia (4) } \\
\text { both ( } 8) \\
\text { yellow discoloration of the ischemic } \\
\text { bowel }\end{array}$ & $\begin{array}{l}\text { resection of the ischemic } \\
\text { segment } \\
\text { abdomen left open }+ \\
\text { second look (14) }\end{array}$ & $\begin{array}{l}\text { Recovery }(10) \\
\text { Death (10) }\end{array}$ \\
\hline Qayed E [21] & $2(878) ; 0.22 \%$ & NA & NA & NA & NA & NA & $\begin{array}{c}\text { diffuse colonic ischemia (1) } \\
\text { Small + large bowel ischemia and } \\
\text { pneumatosis (1) }\end{array}$ & $\begin{array}{l}\text { Total colectomy (1) } \\
\text { Extensive resection (1) }\end{array}$ & $\begin{array}{l}\text { Recovery (1) } \\
\text { Death (1) }\end{array}$ \\
\hline
\end{tabular}

NA: not acknowledged; MAT: mesenteric artery thrombosis; SMA: superior mesenteric artery. 
Table 2. Case reports and case series presenting gastrointestinal ischemia in hospitalized COVID-19 patients under anticoagulant medication.

\begin{tabular}{|c|c|c|c|c|c|c|c|c|c|c|c|c|c|c|c|c|}
\hline Article & Sex & Age & Comorbidities & $\begin{array}{c}\text { Time from } \\
\text { COVID-19 } \\
\text { Diagnosis; } \\
\text { Time from } \\
\begin{array}{c}\text { Admission } \\
\text { (Days) }\end{array} \\
\end{array}$ & $\begin{array}{l}\text { ICU; } \\
\text { Type of } \\
\text { Ventila- } \\
\text { tion }\end{array}$ & $\begin{array}{l}\text { Clinical } \\
\text { Signs at } \\
\text { Presenta- } \\
\text { tion }\end{array}$ & $\begin{array}{l}\text { Leukocytes } \\
\left(/ \mathrm{mm}^{3}\right)\end{array}$ & $\begin{array}{l}\text { CRP } \\
\text { (mg/L) }\end{array}$ & $\begin{array}{c}\text { Lactat } \\
\mathrm{mmol} / \mathrm{L}\end{array}$ & $\begin{array}{l}\text { Ferritin } \\
(\mathrm{ng} / \mathrm{mL})\end{array}$ & $\begin{array}{l}\text { LDH } \\
\text { (U/L) }\end{array}$ & $\begin{array}{l}\text { Thrombocytes } \\
(/ \mathrm{mm} 3)\end{array}$ & $\begin{array}{l}\text { D-Dimers } \\
\text { (ng/mL) }\end{array}$ & Abdominal CT Signs & Treatment & Outcome \\
\hline $\begin{array}{l}\text { Azouz } \\
\mathrm{E}[22]\end{array}$ & $\mathrm{M}$ & 56 & none & $\begin{array}{l}1 ; 2 \\
\text { (hospitalized } \\
\text { for acute } \\
\text { ischemic } \\
\text { stroke) }\end{array}$ & No info & $\begin{array}{l}\text { abdominal } \\
\text { pain and } \\
\text { vomiting }\end{array}$ & No info & - & - & - & - & - & - & $\begin{array}{l}\text { Multiple arterial } \\
\text { thromboembolic } \\
\text { complications: AMS, } \\
\text { right middle cerebral } \\
\text { artery, a free-floating } \\
\text { clot in the aortic arch }\end{array}$ & $\begin{array}{l}\text { Anticoagulation (no } \\
\text { details), endovascular } \\
\text { thrombectomy } \\
\text { Laparotomy + } \\
\text { resection of necrotic } \\
\text { small bowel loops }\end{array}$ & No info \\
\hline $\begin{array}{c}\mathrm{Al} \\
\text { Mahruqi } \\
\mathrm{G}[23]\end{array}$ & $\mathrm{M}$ & 51 & none & $26 ; 24$ & $\begin{array}{l}\text { yes, in- } \\
\text { tubated }\end{array}$ & $\begin{array}{l}\text { Fever, } \\
\text { metabolic } \\
\text { acidosis, } \\
\text { required } \\
\text { inotropes }\end{array}$ & 30,000 & - & 7 & 687 & - & - & 2.5 & $\begin{array}{l}\text { Non-occlusive AMI } \\
\text { Hypoperfused small } \\
\text { bowel, permeable } \\
\text { aorta, SMA, IMA + } \\
\text { deep lower limb } \\
\text { thrombosis }\end{array}$ & $\begin{array}{l}\text { enoxaparin } \\
40 \mathrm{mg} / \text { day from } \\
\text { admission; surgery } \\
\text { refused by family }\end{array}$ & death \\
\hline $\begin{array}{l}\text { Ucpinar } \\
\text { BA [24] }\end{array}$ & $\mathrm{F}$ & 82 & $\begin{array}{l}\text { Atrial } \\
\text { fibrillation, } \\
\text { hyperten- } \\
\text { sion, chronic } \\
\text { kidney } \\
\text { disease }\end{array}$ & $3 ; 3$ & no & - & 14,800 & 196 & 5.1 & - & - & - & 1600 & $\begin{array}{l}\text { SMA thrombosis; } \\
\text { distended small } \\
\text { bowel, with diffuse } \\
\text { submucosal } \\
\text { pneumatosis } \\
\text { portomesenteric gas }\end{array}$ & $\begin{array}{l}\text { fluid resuscitation; } \\
\text { continued ceftriaxone, } \\
\text { enoxaparin 0.4cc } \\
\text { twice daily; } \\
\text { not operable due to } \\
\text { fulminant evolution }\end{array}$ & Death \\
\hline $\begin{array}{l}\text { Karna } \\
\text { ST [25] }\end{array}$ & $\mathrm{F}$ & 61 & $\begin{array}{l}\text { DM, hyper- } \\
\text { tension }\end{array}$ & $4 ; 4$ & $\begin{array}{l}\text { Yes, } \\
\text { HFNO }\end{array}$ & $\begin{array}{c}\text { diffuse } \\
\text { abdominal } \\
\text { pain with } \\
\text { distention }\end{array}$ & 21,400 & 421.6 & 1.4 & - & - & 464,000 & No & $\begin{array}{l}\text { thrombosis of the } \\
\text { distal SMA with } \\
\text { dilated jejunoileal } \\
\text { loops and normal } \\
\text { enhancing bowel wall. }\end{array}$ & $\begin{array}{l}\text { Iv heparin } 5000 \text { ui, } \\
\text { followed by } 1000 \text { ui, } \\
\text { Ecospin and } \\
\text { clopidogrel } \\
\text { Laparotomy after } \\
10 \text { days with } \\
\text { segmental } \\
\text { enterectomy of the } \\
\text { necrotic bowel }\end{array}$ & $\begin{array}{c}\text { Death by } \\
\text { septic } \\
\text { shock and } \\
\text { acute } \\
\text { renal } \\
\text { failure }\end{array}$ \\
\hline $\begin{array}{c}\text { Singh B } \\
{[26]}\end{array}$ & $\mathrm{F}$ & 82 & $\begin{array}{l}\text { Hypertension, } \\
\text { T2DM }\end{array}$ & $32 ; 18$ & $\begin{array}{l}\text { Yes, } \\
\text { Ventila- } \\
\text { tor } \\
\text { support }\end{array}$ & $\begin{array}{c}\text { severe } \\
\text { diffuse } \\
\text { abdominal } \\
\text { distension } \\
\text { and ten- } \\
\text { derness }\end{array}$ & 22,800 & 308 & 2.5 & 136 & 333 & 146,000 & 1.3 & $\begin{array}{l}\text { SMA-colic arteries } \\
\text { thrombosis } \\
\text { pneumatosis } \\
\text { intestinalis affecting } \\
\text { the ascending colon } \\
\text { and cecum }\end{array}$ & $\begin{array}{l}\text { laparotomy, ischemic } \\
\text { colon resection, } \\
\text { ileostomy; heparin in } \\
\text { therapeutic doses pre- } \\
\text { and post-surgery }\end{array}$ & $\begin{array}{l}\text { slow } \\
\text { recovery }\end{array}$ \\
\hline
\end{tabular}


Table 2. Cont.

\begin{tabular}{|c|c|c|c|c|c|c|c|c|c|c|c|c|c|c|c|c|}
\hline Article & Sex & Age & Comorbidities & $\begin{array}{c}\text { Time from } \\
\text { COVID-19 } \\
\text { Diagnosis; } \\
\text { Time from } \\
\text { Admission } \\
\text { (Days) }\end{array}$ & $\begin{array}{l}\text { ICU; } \\
\text { Type of } \\
\text { Ventila- } \\
\text { tion }\end{array}$ & $\begin{array}{l}\text { Clinical } \\
\text { Signs at } \\
\text { Presenta- } \\
\quad \text { tion }\end{array}$ & $\begin{array}{l}\text { Leukocytes } \\
\left(/ \mathrm{mm}^{3}\right)\end{array}$ & $\begin{array}{l}\text { CRP } \\
(\mathrm{mg} / \mathrm{L})\end{array}$ & $\begin{array}{c}\text { Lactat } \\
\mathrm{mmol} / \mathrm{L}\end{array}$ & $\begin{array}{l}\text { Ferritin } \\
\text { (ng/mL) }\end{array}$ & $\begin{array}{l}\text { LDH } \\
\text { (U/L) }\end{array}$ & $\begin{array}{c}\text { Thrombocytes } \\
(/ \mathrm{mm} 3)\end{array}$ & $\begin{array}{l}\text { D-Dimers } \\
\text { (ng/mL) }\end{array}$ & Abdominal CT Signs & Treatment & Outcome \\
\hline $\begin{array}{l}\text { Dinoto } \\
\mathrm{E}[28]\end{array}$ & $\mathrm{F}$ & 84 & $\begin{array}{l}\text { DM, hyper- } \\
\text { tension, } \\
\text { renal failure }\end{array}$ & $2 ; 2$ & no & $\begin{array}{l}\text { Acute } \\
\text { abdominal } \\
\text { pain and } \\
\text { disten- } \\
\text { sion; }\end{array}$ & 18,000 & 32.47 & - & - & 431 & - & 6937 & $\begin{array}{l}\text { SMA origin stenosis } \\
\text { and occlusion at } 2 \mathrm{~cm} \\
\text { from the origin, } \\
\text { absence of bowel } \\
\text { enhancement }\end{array}$ & $\begin{array}{c}\text { Endovascular } \\
\text { thrombectomy of } \\
\text { SMA; } \\
\text { surgical transfemoral } \\
\text { thrombectomy and } \\
\text { distal superficial } \\
\text { femoral artery } \\
\text { stenting }\end{array}$ & $\begin{array}{l}\text { Death due } \\
\text { to respira- } \\
\text { tory } \\
\text { failure }\end{array}$ \\
\hline
\end{tabular}


Table 3. Case reports and case series presenting gastrointestinal ischemia in non-hospitalized COVID-19 patients.

\begin{tabular}{|c|c|c|c|c|c|c|c|c|c|c|c|c|c|c|c|}
\hline Article & Sex & Age & Comorbidities & $\begin{array}{c}\text { Time from } \\
\text { COVID-19 } \\
\text { Diagnosis } \\
\text { (Days) }\end{array}$ & $\begin{array}{l}\text { Clinical } \\
\text { Signs at } \\
\text { Presenta- } \\
\text { tion }\end{array}$ & $\begin{array}{l}\text { Leukocyte } \\
\text { Count } \\
\left(/ \mathrm{mm}^{3}\right)\end{array}$ & $\underset{(\mathrm{mg} / \mathrm{L})}{\mathrm{CRP}}$ & $\begin{array}{l}\text { Lactate } \\
\mathrm{mmol} / \mathrm{L}\end{array}$ & $\begin{array}{l}\text { Ferritin } \\
(\mathrm{ng} / \mathrm{mL})\end{array}$ & LDH (U/L) & $\begin{array}{c}\text { Thrombocytes } \\
\left(/ \mathrm{mm}^{3}\right)\end{array}$ & $\begin{array}{l}\text { D-Dimers } \\
\text { (ng/mL) }\end{array}$ & $\begin{array}{c}\text { Abdominal CT } \\
\text { Signs }\end{array}$ & Treatment & Outcome \\
\hline $\begin{array}{c}\text { Sevella, P } \\
{[30]}\end{array}$ & M & 44 & none & 10 & $\begin{array}{l}\text { Acute } \\
\text { abdominal } \\
\text { pain consti- } \\
\text { pation, } \\
\text { vomiting }\end{array}$ & 23,400 & - & - & - & 1097 & 360,000 & 1590 & $\begin{array}{c}\text { Viable jejunum, } \\
\text { ischemic bowel, } \\
\text { peritoneal } \\
\text { thickening with fat } \\
\text { stranding; free fluid } \\
\text { in the peritoneal } \\
\text { cavity }\end{array}$ & $\begin{array}{l}\text { LMWH } 60 \mathrm{mg} \text { daily } \\
\text { Piperacillin } 4 \mathrm{~g} / \text { day } \\
\text { Tazobactam } \\
500 \mathrm{mg} / \text { day } \\
\text { Extensive small bowel } \\
\text { + right colon resection }\end{array}$ & death \\
\hline $\begin{array}{c}\text { Nasseh S } \\
\text { [31] }\end{array}$ & $\mathrm{M}$ & 68 & no info & $\begin{array}{c}\text { First } \\
\text { diagnosis }\end{array}$ & $\begin{array}{l}\text { epigastric } \\
\text { pain and } \\
\text { diarrhea } \\
\text { for } 4 \text { days }\end{array}$ & 17,660 & 125 & - & - & - & - & 6876 & $\begin{array}{l}\text { terminal segment of } \\
\text { the ileocolic artery } \\
\text { thrombosis; } \\
\text { thickening of the } \\
\text { right colon wall and } \\
\text { the last } 30 \mathrm{~cm} \text { of the } \\
\text { small bowl }\end{array}$ & $\begin{array}{l}\text { unfractionated heparin } \\
\text { laparoscopy -no bowel } \\
\text { resection needed }\end{array}$ & recovery \\
\hline$\underset{[32]}{\operatorname{Aleman} W}$ & M & 44 & none & 20 & $\begin{array}{l}\text { severe ab- } \\
\text { dominopelvic } \\
\text { pain }\end{array}$ & 36,870 & - & - & 456.23 & - & 574,000 & 263.87 & $\begin{array}{l}\text { absence of flow at } \\
\text { SMV, splenic, portal } \\
\text { vein; } \\
\text { Small bowel loop } \\
\text { dilatation and } \\
\text { mesenteric fat } \\
\text { edema }\end{array}$ & $\begin{array}{l}\text { enoxaparin and pain } \\
\text { control medication } \\
6 \text { days, then switched } \\
\text { to warfarin } 6 \text { months }\end{array}$ & recovery \\
\hline $\begin{array}{c}\text { Randhawa } \\
\mathrm{J}[34]\end{array}$ & $\mathrm{F}$ & 62 & none & $\begin{array}{c}\text { First } \\
\text { diagnosis }\end{array}$ & $\begin{array}{l}\text { right upper } \\
\text { quadrant } \\
\text { pain and } \\
\text { loss of } \\
\text { appetite for } \\
\text { 14 days }\end{array}$ & $\begin{array}{l}\text { Normal } \\
\text { limits }\end{array}$ & - & - & - & 346 & - & - & $\begin{array}{l}\text { large thrombus } \\
\text { involving the SMV, } \\
\text { the main portal vein } \\
\text { with extension into } \\
\text { its branches }\end{array}$ & $\begin{array}{l}\text { Fondaparinux } \\
\text { 2.5. mg } 5 \text { days, then } \\
\text { warfarin } 4 \mathrm{mg} \\
\text { (adjusted by INR), } \\
6 \text { months }\end{array}$ & recovery \\
\hline $\begin{array}{c}\text { Cheung S } \\
\text { [35] }\end{array}$ & $\mathrm{M}$ & 55 & none & $\begin{array}{c}12 \\
\text { (discharged } \\
\text { for } 7 \text { days) }\end{array}$ & $\begin{array}{l}\text { Nausea, } \\
\text { vomiting } \\
\text { and } \\
\text { worsening } \\
\text { generalized } \\
\text { abdominal } \\
\text { pain with } \\
\text { guarding }\end{array}$ & 12,446 & - & 0.68 & - & - & - & - & $\begin{array}{l}\text { low-density clot, } \\
1.6 \mathrm{~cm} \text { in length, } \\
\text { causing high-grade } \\
\text { narrowing of the } \\
\text { proximal SMA }\end{array}$ & $\begin{array}{l}\text { continuous heparin } \\
\text { infusion continued } 8 \mathrm{~h} \\
\text { postoperative, } \\
\text { Laparotomy with SMA } \\
\text { thromboembolectomy } \\
\text { and enterectomy } \\
\text { (small bowel) }\end{array}$ & recovery \\
\hline
\end{tabular}


Table 3. Cont.

\begin{tabular}{|c|c|c|c|c|c|c|c|c|c|c|c|c|c|c|c|}
\hline Article & Sex & Age & Comorbidities & $\begin{array}{c}\text { Time from } \\
\text { COVID-19 } \\
\text { Diagnosis } \\
\text { (Days) }\end{array}$ & $\begin{array}{l}\text { Clinical } \\
\text { Signs at } \\
\text { Presenta- } \\
\text { tion }\end{array}$ & $\begin{array}{c}\text { Leukocyte } \\
\text { Count } \\
\left(/ \mathrm{mm}^{3}\right)\end{array}$ & $\begin{array}{c}\text { CRP } \\
\text { (mg/L) }\end{array}$ & $\begin{array}{l}\text { Lactate } \\
\mathrm{mmol} / \mathrm{L}\end{array}$ & $\begin{array}{l}\text { Ferritin } \\
(\mathrm{ng} / \mathrm{mL})\end{array}$ & LDH (U/L) & $\begin{array}{c}\text { Thrombocytes } \\
\left(/ \mathrm{mm}^{3}\right)\end{array}$ & $\begin{array}{l}\text { D-Dimers } \\
\text { (ng/mL) }\end{array}$ & $\underset{\text { Signs }}{\text { Abdominal CT }}$ & Treatment & Outcome \\
\hline $\begin{array}{c}\text { Beccara L } \\
\text { [36] }\end{array}$ & M & 52 & none & $\begin{array}{l}22 \text { ( } 5 \text { days } \\
\text { after } \\
\text { discharge and } \\
\text { cessation } \\
\text { prophylactic } \\
\text { LWMH) }\end{array}$ & $\begin{array}{l}\text { vomiting } \\
\text { and } \\
\text { abdominal } \\
\text { pain, } \\
\text { tenderness } \\
\text { in epigas- } \\
\text { trium and } \\
\text { mesogas- } \\
\text { trium }\end{array}$ & 30,000 & 222 & - & - & - & - & - & $\begin{array}{l}\text { arterial thrombosis } \\
\text { of vessels efferent of } \\
\text { the SMA with bowel } \\
\text { distension }\end{array}$ & $\begin{array}{l}\text { Enterectomy (small } \\
\text { bowel) } \\
\text { LMWH plus aspirin } \\
100 \mathrm{mg} / \text { day at } \\
\text { discharge }\end{array}$ & recovery \\
\hline $\begin{array}{l}\text { Vulliamy P } \\
{[37]}\end{array}$ & $\mathrm{M}$ & 75 & none & 14 & $\begin{array}{l}\text { abdominal } \\
\text { pain and } \\
\text { vomiting } \\
\text { for } 2 \text { days }\end{array}$ & 18,100 & 3.2 & - & - & - & 497,000 & 320 & $\begin{array}{l}\text { intraluminal } \\
\text { thrombus was } \\
\text { present in the } \\
\text { descending thoracic } \\
\text { aorta with embolic } \\
\text { occlusion of SMA }\end{array}$ & $\begin{array}{l}\text { Catheter-directed } \\
\text { thrombolysis, } \\
\text { enterectomy (small } \\
\text { bowel) }\end{array}$ & recovery \\
\hline $\begin{array}{c}\text { De Barry O } \\
{[38]}\end{array}$ & $\mathrm{F}$ & 79 & none & $\begin{array}{c}\text { First } \\
\text { diagnosis }\end{array}$ & $\begin{array}{l}\text { Epigastric } \\
\text { pain, } \\
\text { diarrhea, } \\
\text { fever for } 8 \\
\text { days, acute } \\
\text { dyspnea }\end{array}$ & 12600 & 125 & 5.36 & - & - & - & - & $\begin{array}{l}\text { SMV, portal vein, } \\
\text { SMA, and jejunal } \\
\text { artery thrombosis } \\
\text { Distended loops, } \\
\text { free fluid }\end{array}$ & $\begin{array}{c}\text { anticoagulation } \\
\text { Resection of affected } \\
\text { colon+ ileum, SMA } \\
\text { thrombolysis, } \\
\text { thrombectomy }\end{array}$ & death \\
\hline $\begin{array}{c}\text { Posada } \\
\text { Arango } \\
{[40]}\end{array}$ & $\begin{array}{c}\mathrm{M} \\
\mathrm{F} \\
\mathrm{F}\end{array}$ & $\begin{array}{l}62 \\
22 \\
65\end{array}$ & $\begin{array}{l}\text { None } \\
\text { Appendectomy } \\
7 \text { days } \\
\text { before } \\
\text { left } \\
\text { nephrec- } \\
\text { tomy, }\end{array}$ & $\begin{array}{c}5 \\
3 \\
15\end{array}$ & $\begin{array}{c}\text { colicative } \\
\text { abdominal } \\
\text { pain at } \\
\text { food intake; } \\
\text { unsystem- } \\
\text { atized } \\
\text { gastroin- } \\
\text { testinal } \\
\text { symptoms; } \\
\text { abdominal } \\
\text { pain in the } \\
\text { upper } \\
\text { hemiab- } \\
\text { domen }\end{array}$ & $\begin{array}{c}20,100 \\
- \\
-\end{array}$ & $\begin{array}{l}- \\
-\end{array}$ & $\begin{array}{l}- \\
- \\
-\end{array}$ & $\begin{array}{c}1536 \\
- \\
-\end{array}$ & $\begin{array}{c}534 \\
- \\
-\end{array}$ & $\begin{array}{l}- \\
-\end{array}$ & $\begin{array}{l}- \\
- \\
-\end{array}$ & $\begin{array}{l}\text { Case 1: thrombus in } \\
\text { distal SMA and its } \\
\text { branches, intestinal } \\
\text { loops dilatation, } \\
\text { hydroaerical levels, } \\
\text { free fluid } \\
\text { thrombosis of SMV } \\
\text { Case 2: SMV } \\
\text { thrombosis and } \\
\text { adiacent fat edema } \\
\text { Case 3: thrombi in } \\
\text { the left jejunal artery } \\
\text { branch with } \\
\text { infarction of the } \\
\text { corresponding } \\
\text { jejunal loops }\end{array}$ & $\begin{array}{l}\text { Case 1: Laparotomy: } \\
\text { extensive jejunum + } \\
\text { ileum ischemia; } \\
\text { surgery could not be } \\
\text { performed } \\
\text { Case 2: } \\
\text { Anticoagulation } \\
\text { analgesic and } \\
\text { antibiotics } \\
\text { Case 3: segmental } \\
\text { enterectomy }\end{array}$ & $\begin{array}{l}\text { Case 1: } \\
\text { death } \\
\text { Case 2: } \\
\text { recovery } \\
\text { Case 3: } \\
\text { recovery }\end{array}$ \\
\hline
\end{tabular}


Table 3. Cont.

\begin{tabular}{|c|c|c|c|c|c|c|c|c|c|c|c|c|c|c|c|}
\hline Article & Sex & Age & Comorbidities & $\begin{array}{c}\text { Time from } \\
\text { COVID-19 } \\
\text { Diagnosis } \\
\text { (Days) }\end{array}$ & $\begin{array}{l}\text { Clinical } \\
\text { Signs at } \\
\text { Presenta- } \\
\text { tion }\end{array}$ & $\begin{array}{l}\text { Leukocyte } \\
\text { Count } \\
\left(/ \mathrm{mm}^{3}\right)\end{array}$ & $\begin{array}{l}\text { CRP } \\
\text { (mg/L) }\end{array}$ & $\begin{array}{l}\text { Lactate } \\
\mathrm{mmol} / \mathrm{L}\end{array}$ & $\begin{array}{l}\text { Ferritin } \\
\text { (ng/mL) }\end{array}$ & LDH (U/L) & $\begin{array}{l}\text { Thrombocytes } \\
\left(/ \mathrm{mm}^{3}\right)\end{array}$ & $\begin{array}{l}\text { D-Dimers } \\
\text { (ng/mL) }\end{array}$ & $\begin{array}{c}\text { Abdominal CT } \\
\text { Signs }\end{array}$ & Treatment & Outcome \\
\hline $\begin{array}{l}\text { Pang JHQ } \\
{[41]}\end{array}$ & $\mathrm{M}$ & 30 & none & $\begin{array}{c}\text { First } \\
\text { diagnosis }\end{array}$ & $\begin{array}{l}\text { colicky } \\
\text { abdominal } \\
\text { pain, } \\
\text { vomiting }\end{array}$ & - & - & - & - & - & - & 20 & $\begin{array}{l}\text { SMV thrombosis } \\
\text { with diffuse mural } \\
\text { thickening and fat } \\
\text { stranding of } \\
\text { multiple jejunal } \\
\text { loops }\end{array}$ & $\begin{array}{c}\text { conservative, } \\
\text { anticoagulation with } \\
\text { LMWH 1mg/kc, twice } \\
\text { daily, } 3 \text { months; } \\
\text { readmitted and } \\
\text { operated for congenital } \\
\text { adherence causing } \\
\text { small bowel } \\
\text { obstruction }\end{array}$ & recovery \\
\hline Lari E [42] & $\mathrm{M}$ & 38 & none & $\begin{array}{c}\text { First } \\
\text { diagnosis }\end{array}$ & $\begin{array}{l}\text { abdominal } \\
\text { pain, } \\
\text { nausea, } \\
\text { intractable } \\
\text { vomiting, } \\
\text { and } \\
\text { shortness } \\
\text { of breath } \\
\end{array}$ & $\begin{array}{l}\text { Mild leuko- } \\
\text { cytosis }\end{array}$ & - & 2.2 & - & - & - & 2100 & $\begin{array}{l}\text { extensive } \\
\text { thrombosis of the } \\
\text { portal, splenic, } \\
\text { superior, and } \\
\text { inferior mesenteric } \\
\text { vein + mild bowel } \\
\text { ischemia }\end{array}$ & $\begin{array}{l}\text { Anticoagulation, } \\
\text { resection of the } \\
\text { affected bowel loop }\end{array}$ & No info \\
\hline $\begin{array}{c}\text { Carmo } \\
\text { Filho A [43] }\end{array}$ & $\mathrm{M}$ & 33 & $\begin{array}{l}\text { Obesity } \\
\text { (BMI: 33), } \\
\text { other not } \\
\text { reported }\end{array}$ & 7 & $\begin{array}{l}\text { severe low } \\
\text { back pain } \\
\text { radiating to } \\
\text { the hy- } \\
\text { pogastric } \\
\text { region }\end{array}$ & - & 58.2 & - & 1570 & - & - & 879 & $\begin{array}{l}\text { enlarged inferior } \\
\text { mesenteric vein not } \\
\text { filled by contrast } \\
\text { associated with } \\
\text { infiltration of the } \\
\text { adjacent adipose } \\
\text { planes }\end{array}$ & $\begin{array}{l}\text { enoxaparin } 5 \text { days, } \\
\text { followed by long term } \\
\text { oral warfarin }\end{array}$ & recovery \\
\hline $\begin{array}{l}\text { Hanif M } \\
{[44]}\end{array}$ & $\mathrm{F}$ & 20 & none & 8 & $\begin{array}{l}\text { abdominal } \\
\text { pain and } \\
\text { abdominal } \\
\text { distension }\end{array}$ & 15,900 & 62 & - & 1435.3 & 825 & 633,000 & 2340 & not performed & $\begin{array}{l}\text { evidence of SMA } \\
\text { thrombosis; } \\
\text { enterectomy with } \\
\text { exteriorization of both } \\
\text { ends }\end{array}$ & recovery \\
\hline $\begin{array}{l}\text { Amaravathi } \\
\text { U [45] }\end{array}$ & $\mathrm{M}$ & 45 & none & 5 & $\begin{array}{l}\text { Acute } \\
\text { epigastric } \\
\text { and peri- } \\
\text { umbilical } \\
\text { pain }\end{array}$ & - & $\begin{array}{l}\text { Normal } \\
\text { value }\end{array}$ & 1.3 & 324.3 & - & - & 5.3 & $\begin{array}{l}\text { SMA and SMV } \\
\text { thrombus }\end{array}$ & $\begin{array}{c}\text { i.v. heparin; } \\
\text { Laparotomy with SMA } \\
\text { thrombectomy; } \\
48 \mathrm{~h} \text { Second look: } \\
\text { resection of the } \\
\text { gangrenous bowel } \\
\text { segment }\end{array}$ & No info \\
\hline $\begin{array}{l}\text { Al Mahruqi } \\
\text { G [23] }\end{array}$ & $\mathrm{M}$ & 51 & none & 4 & $\begin{array}{c}\text { generalized } \\
\text { abdominal } \\
\text { pain, } \\
\text { nausea, } \\
\text { vomiting }\end{array}$ & 16,000 & - & - & 619 & - & - & 10 & $\begin{array}{l}\text { SMA thrombosis } \\
\text { and non-enhancing } \\
\text { proximal ileal loops } \\
\text { consistent with small } \\
\text { bowel ischemia }\end{array}$ & $\begin{array}{l}\text { unfractionated heparin, } \\
\text { thrombectomy + } \\
\text { repeated resections of } \\
\text { the ischemic bowel at } \\
\text { relook (je- } \\
\text { junum+ileon+cecum) }\end{array}$ & $\begin{array}{l}\text { Case } 2 \text { : } \\
\text { recovery }\end{array}$ \\
\hline
\end{tabular}


Table 3. Cont.

\begin{tabular}{|c|c|c|c|c|c|c|c|c|c|c|c|c|c|c|c|}
\hline Article & Sex & Age & Comorbidities & $\begin{array}{c}\text { Time from } \\
\text { COVID-19 } \\
\text { Diagnosis } \\
\text { (Days) }\end{array}$ & $\begin{array}{c}\text { Clinical } \\
\text { Signs at } \\
\text { Presenta- } \\
\text { tion }\end{array}$ & $\begin{array}{l}\text { Leukocyte } \\
\text { Count } \\
\left(/ \mathrm{mm}^{3}\right)\end{array}$ & $\begin{array}{c}\text { CRP } \\
\text { (mg/L) }\end{array}$ & $\begin{array}{l}\text { Lactate } \\
\mathrm{mmol} / \mathrm{L}\end{array}$ & $\begin{array}{l}\text { Ferritin } \\
\text { (ng/mL) }\end{array}$ & LDH (U/L) & $\begin{array}{l}\text { Thrombocytes } \\
\left(/ \mathrm{mm}^{3}\right)\end{array}$ & $\begin{array}{l}\text { D-Dimers } \\
\text { (ng/mL) }\end{array}$ & $\underset{\text { Signs }}{\text { Abdominal CT }}$ & Treatment & Outcome \\
\hline $\begin{array}{l}\text { Goodfellow } \\
\text { M [46] }\end{array}$ & $\mathrm{F}$ & 36 & $\begin{array}{l}\text { RYGB, } \\
\text { depression, } \\
\text { asthma }\end{array}$ & 6 & $\begin{array}{c}\text { epigastric } \\
\text { pain, } \\
\text { irradiating } \\
\text { back, } \\
\text { nausea }\end{array}$ & 9650 & 1.2 & 0.7 & - & - & - & - & $\begin{array}{c}\text { abrupt cut-off of the } \\
\text { SMV in the proximal } \\
\text { portion; } \\
\text { diffuse infiltration of } \\
\text { the mesentery, wall } \\
\text { thickening of small } \\
\text { bowel }\end{array}$ & $\begin{array}{l}\text { IV heparin infusion, } \\
\text { followed by } 18,000 \mathrm{UI} \\
\text { delteparin after } 72 \mathrm{~h}\end{array}$ & recovery \\
\hline $\begin{array}{l}\text { Abeysekera } \\
\text { KW [26] }\end{array}$ & M & 42 & Hepatitis B & 14 & $\begin{array}{l}\text { right } \\
\text { hypochon- } \\
\text { drial pain, } \\
\text { progres- } \\
\text { sively } \\
\text { increasing } \\
\text { for } 9 \text { days }\end{array}$ & - & - & - & - & - & - & - & $\begin{array}{l}\text { enhancement of the } \\
\text { entire length of the } \\
\text { portal vein and a } \\
\text { smaller thrombus in } \\
\text { the mid-superior } \\
\text { mesenteric vein, } \\
\text { mural edema of the } \\
\text { distal duodenum, } \\
\text { distal small bowel, } \\
\text { and descending } \\
\text { colon }\end{array}$ & $\begin{array}{c}\text { factor Xa inhibitor } \\
\text { apixaban } 5 \mathrm{mg} \times 2 / \text { day, } \\
6 \mathrm{months}\end{array}$ & $\begin{array}{l}- \\
\text { recovery }\end{array}$ \\
\hline $\begin{array}{l}\text { Rodriguez- } \\
\text { Nakamura } \\
\text { RM [27] }\end{array}$ & $\begin{array}{l}\mathrm{M} \\
\mathrm{F}\end{array}$ & $\begin{array}{l}45 \\
42\end{array}$ & $\begin{array}{l}\text {-vitiligo } \\
\text {-obesity }\end{array}$ & 14 & $\begin{array}{c}\text { severe } \\
\text { mesogas- } \\
\text { tric pain, } \\
\text { nausea, } \\
\text { diaphoresis }\end{array}$ & $\begin{array}{l}16,400 \\
18,800\end{array}$ & $\begin{array}{l}367 \\
239\end{array}$ & - & $\begin{array}{c}970 \\
-\end{array}$ & - & $\begin{array}{c}685,000 \\
-\end{array}$ & $\begin{array}{c}1450 \\
14,407\end{array}$ & $\begin{array}{l}\text { Case 1: SMI of } \\
\text { thrombotic etiology } \\
\text { with partial } \\
\text { rechanneling } \\
\text { through the middle } \\
\text { colic artery, and } \\
\text { hypoxic-ischemic } \\
\text { changes in the distal } \\
\text { ileum and the cecum } \\
\text { Case 2: thrombosis } \\
\text { of the portal and } \\
\text { mesenteric veins and } \\
\text { an abdominopelvic } \\
\text { collection in the } \\
\text { mesentery with gas }\end{array}$ & $\begin{array}{l}\text { Case 1: resection with } \\
\text { entero-enteral } \\
\text { anastomosis; } \\
\text { rivaroxaban } \\
10 \text { mg/day, } 6 \text { months } \\
\text { Case 2: Loop resection, } \\
\text { entero-enteral manual } \\
\text { anastomosis, partial } \\
\text { omentectomy, and } \\
\text { cavity wash (fecal } \\
\text { peritonitis) }\end{array}$ & $\begin{array}{c}\text { Case 1: } \\
\text { Recovery } \\
\text { Case 2: } \\
\text { death }\end{array}$ \\
\hline Plotz B [47] & $\mathrm{F}$ & 27 & $\begin{array}{l}\text { SLE with } \\
\text { ITP }\end{array}$ & $\begin{array}{c}\text { First } \\
\text { diagnosis }\end{array}$ & $\begin{array}{l}\text { acute onset } \\
\text { nausea, } \\
\text { vomiting, } \\
\text { and } \\
\text { non-bloody } \\
\text { diarrhea }\end{array}$ & - & - & - & - & - & - & 5446 & $\begin{array}{l}\text { diffuse small bowel } \\
\text { edema }\end{array}$ & $\begin{array}{l}\text { enoxaparin, long term } \\
\text { apixaban at discharge }\end{array}$ & recovery \\
\hline
\end{tabular}


Table 3. Cont.

\begin{tabular}{|c|c|c|c|c|c|c|c|c|c|c|c|c|c|c|c|}
\hline Article & Sex & Age & Comorbidities & $\begin{array}{c}\text { Time from } \\
\text { COVID-19 } \\
\text { Diagnosis } \\
\text { (Days) }\end{array}$ & $\begin{array}{l}\text { Clinical } \\
\text { Signs at } \\
\text { Presenta- } \\
\quad \text { tion }\end{array}$ & $\begin{array}{l}\text { Leukocyte } \\
\text { Count } \\
\left(/ \mathrm{mm}^{3}\right)\end{array}$ & $\underset{(\mathrm{mg} / \mathrm{L})}{\mathrm{CRP}}$ & $\begin{array}{l}\text { Lactate } \\
\mathrm{mmol} / \mathrm{L}\end{array}$ & $\begin{array}{l}\text { Ferritin } \\
(\mathrm{ng} / \mathrm{mL})\end{array}$ & LDH (U/L) & $\begin{array}{c}\text { Thrombocytes } \\
\left(/ \mathrm{mm}^{3}\right)\end{array}$ & $\begin{array}{l}\text { D-Dimers } \\
\text { (ng/mL) }\end{array}$ & $\begin{array}{l}\text { Abdominal CT } \\
\text { Signs }\end{array}$ & Treatment & Outcome \\
\hline $\begin{array}{c}\text { Chiu CY } \\
{[48]}\end{array}$ & F & 49 & $\begin{array}{c}\text { Hypertension, } \\
\text { DM, } \\
\text { chronic } \\
\text { kidney } \\
\text { disease }\end{array}$ & 28 & $\begin{array}{c}\text { diffuse } \\
\text { abdominal } \\
\text { pain } \\
\text { melena and } \\
\text { hemateme- } \\
\text { sis }\end{array}$ & - & - & - & - & - & - & 12,444 & $\begin{array}{l}\text { distended proximal } \\
\text { jejunum with mural } \\
\text { thickening }\end{array}$ & $\begin{array}{l}\text { laparotomy, proximal } \\
\text { jejunum resection }\end{array}$ & no info \\
\hline$\underset{[49]}{\text { Farina D }}$ & M & 70 & no info & 3 & $\begin{array}{l}\text { abdominal } \\
\text { pain, } \\
\text { nausea }\end{array}$ & 15,300 & 149 & - & - & - & - & - & $\begin{array}{l}\text { acute small bowel } \\
\text { hypoperfusion, SMA } \\
\text { thromboembolism }\end{array}$ & $\begin{array}{l}\text { not operable due to } \\
\text { general condition }\end{array}$ & Death \\
\hline
\end{tabular}

SMA: superior mesenteric artery; SMV: superior mesenteric vein; DM: diabetes mellitus; T2DM: type 2 diabetes mellitus; AMI: acute mesenteric ischemia; IMV: inferior mesenteric vein; RYGB: Roux-en-Y gastric bypass (bariatric surgery). 


\subsection{Risk of Bias}

The studies analyzed in the present review were comparable in terms of patient selection, methodology, therapeutic approach, and the report of final outcome. However, there were differences in the reported clinical and laboratory data. The sample size was small, most of them being case reports or case series, which may be a significant source of bias. Therefore, studies were compared only qualitatively.

\section{Results}

After duplication removal, a total of 36 articles were included in the review, reporting data on a total of 89 patients. Among these, we identified 6 retrospective studies [16-21], documenting intestinal ischemia in 55 patients admitted to intensive care units (ICU) with COVID-19 pneumonia for whom surgical consult was necessary (Table 1).

We also identified 30 case reports or case series [22-51] presenting 34 cases of acute bowel ischemia in patients positive for SARS-CoV-2 infection in different clinical settings. 8 cases were previously hospitalized for COVID-19 pneumonia and under anticoagulant medication (Table 2). In 26 cases, the acute ischemic event appeared as the first symptom of COVID-19 disease, or in mild forms treated at home, or after discharge for COVID -19 pneumonia and cessation of the anticoagulant medication (Table 3).

\subsection{Risk Factors of Intestinal Ischemia in COVID-19 Patients}

Out of a total of 89 patients included in the review, $63(70.7 \%)$ were hospitalized for severe forms of COVID-19 pneumonia at the moment of onset. These patients were receiving anticoagulant medication when reported, consisting of low molecular weight heparin (LMWH) at prophylactic doses. The incidence of acute intestinal ischemia in ICU patients with COVID-19 varied widely between $0.22-10.5 \%$ (Table 1 ). In a study by O'Shea et al. [20], 26\% of hospitalized patients for COVID-19 pneumonia who underwent imagistic examination, presented results positive for coagulopathy, and in $22 \%$ of these cases, the thromboembolic events were with multiple locations.

The mean age was 56.9 years. We observed a significantly lower age in non-hospitalized COVID-19 patients presenting with acute intestinal ischemia when compared to the previously hospitalized group $(p<0.0001)$.

There is a slight male to female predominance $(\mathrm{M}: \mathrm{F}=1: 68)$. Obesity might be considered a possible risk factor, with a reported mean BMI of 31.2-32.5 in hospitalized patients $[16,18,19]$. However, this association should be regarded with caution, since obesity is also a risk factor for severe forms of COVID-19. Prolonged stay in intensive care, intubation, and the need for vasopressor medication was associated with increased risk of acute bowel ischemia $[8,18,19]$.

Diabetes mellitus and hypertension were the most frequent comorbidities encountered in case reports ( 8 in 34 patients, 23\%), and 7 out of 8 patients presented both (Table 4 ). There was no information regarding the comorbidities in the retrospective studies included in the review.

\subsection{Clinical Features in COVID-19 Patients with Acute Mesenteric Ischemia}

Abdominal pain, out of proportion to physical findings, is a hallmark of portomesenteric thrombosis, typically associated with fever and leukocytosis [4]. Abdominal pain was encountered in all cases, either generalized from the beginning, of high intensity, or firstly localized in the epigastrium or the mezogastric area. In cases of portal vein thrombosis, the initial location may be in the right hypochondrium, mimicking biliary colic $[26,34]$.

Fever is less useful in COVID-19 infected patients, taking into consideration that fever is a general sign of infection, and on the other hand, these patients might be already under antipyretic medication. 
Table 4. Demographic data of the patients included in the review.

\begin{tabular}{|c|c|}
\hline Nr. of Patients & 89 \\
\hline M & $48(61.5 \%$ *) \\
\hline $\mathrm{F}$ & $30\left(38.5 \%{ }^{*}\right)$ \\
\hline NA & 11 \\
\hline The first sign of COVID-19 & $6(6.7 \%)$ \\
\hline Home treated & $17(19.1 \%)$ \\
\hline $\begin{array}{l}\text { Hospitalized } \\
\text { - ICU }\end{array}$ & $\begin{array}{c}63(70.7 \%) \\
58 \text { (92\% of hospitalized patients) }\end{array}$ \\
\hline Discharged & $3(3.3 \%)$ \\
\hline $\begin{array}{l}\text { Time from diagnosis of COVID-19 infection } \\
\text { - } \quad \text { Non-Hospitalized } \\
\text { - } \quad \text { Hospitalized ( }{ }^{*} \text { when mentioned) }\end{array}$ & $\begin{array}{l}8.7 \pm 7.4(1-28 \text { days }) \\
9.6 \pm 8.3 \text { ( } 1-26 \text { days })\end{array}$ \\
\hline Time from admission in hospitalized patients & 1-104 days \\
\hline $\begin{array}{l}\text { Age }(\text { mean) } \\
\text { - } \quad \text { Hospitalized } \\
\text { - } \quad \text { Non-hospitalized }\end{array}$ & $\begin{array}{c}59.3 \pm 12.7 \text { years } \\
62 \pm 9.6 \text { years. }(p<0.0001) \\
52.8 \pm 16.4 \text { years. }\end{array}$ \\
\hline BMI & $31.2-32.5$ \\
\hline $\begin{array}{ll}\text { Comorbidities } \\
\text { - } & \text { Hypertension } \\
\text { - } & \text { DM } \\
\text { - } & \text { smokers } \\
\text { - } & \text { Atrial fibrillation } \\
\text { - } & \text { COPD } \\
\text { - } & \text { Cirrhosis } \\
\text { - } & \text { RYGB } \\
\text { - } & \text { Vitiligo } \\
\text { - } & \text { Recent appendicitis } \\
\text { - } & \text { Operated gastric cancer } \\
\text { - } & \text { Alzheimer disease } \\
\text { - } & \text { SLE }\end{array}$ & $\begin{array}{l}8 \\
7 \\
2 \\
1 \\
2 \\
1 \\
1 \\
1 \\
1 \\
1 \\
1 \\
1\end{array}$ \\
\hline
\end{tabular}

Other clinical signs reported were nausea, anorexia, vomiting, and food intolerance $[23,31,38,45]$. However, these gastrointestinal signs are encountered in $30-40 \%$ of patients with SARS-CoV-2 infection. In a study by Kaafarani et al., up to half of the patients with gastrointestinal features presented some degrees of intestinal hypomotility, possibly due to direct viral invasion of the enterocytes and neuro-enteral disturbances [16].

Physical exam evidenced abdominal distension, reduced bowel sounds, and tenderness at palpation. Guarding may be evocative for peritonitis due to compromised vascularization of bowel loops and bacterial translocation or franc perforation [35,39].

A challenging case was presented by Goodfellow et al. [25] in a patient with a recent history of bariatric surgery with Roux en Y gastric bypass, presenting with acute abdominal pain which imposed the differential diagnosis with an internal hernia.

Upcinar et al. [24] reported a case of an 82-years female that also associated atrial fibrillation. The patient was anticoagulated with enoxaparin $0.4 \mathrm{cc}$ twice daily before admission and continued the anticoagulant therapy during hospitalization for COVID-19 pneumonia. Bedside echocardiography was performed to exclude atrial thrombus. Although SMA was reported related to COVID-19 pneumonia, atrial fibrillation is a strong risk factor for SMA of non-COVID-19 etiology. 
In ICU patients, acute bowel ischemia should be suspected in cases that present acute onset of digestive intolerance and stasis, abdominal distension, and require an increase of vasopressor medication [19].

\subsection{Imagistic and Lab Test Findings}

D-dimer is a highly sensitive investigation for the prothrombotic state caused by COVID-19 [45] and, when reported, was found to be above the normal values. Leukocytosis and acute phase biomarkers, such as fibrinogen and CRP were elevated, mirroring the intensity of inflammation and sepsis caused by the ischemic bowel. However, there was no significant statistical correlation between either the leukocyte count ( $p=0.803$ ) or D-dimers $(p=0.08)$ and the outcome. Leucocyte count may be within normal values in case of early presentation [34]. Thrombocytosis and thrombocytopenia have been reported in published cases with mesenteric ischemia $[30,35,42,46,50]$.

Lactate levels were reported in 9 cases, with values higher than $2 \mathrm{mmol} / \mathrm{L}$ in 5 cases (55\%). LDH was determined in 6 cases, and it was found to be elevated in all cases, with a mean value of $594+/-305 \mathrm{U} / \mathrm{L}$.

Ferritin is another biomarker of potential value in mesenteric ischemia, that increases due to ischemia-reperfusion cellular damage. In the reviewed studies, serum ferritin was raised in 7 out of 9 reported cases, with values ranging from 456 to $1570 \mathrm{ng} / \mathrm{mL}$. However, ferritin levels were found to be correlated also with the severity of pulmonary lesions in COVID-19 patients [52]. Due to the low number of cases in which lactate, LDH, and ferritin were reported, no statistical association could be performed with the severity of lesions or with adverse outcomes.

The location and extent of venous or arterial thrombosis were determined by contrastenhanced abdominal CT, which also provided important information on the viability of the intestinal segment whose vascularity was affected.

Radiological findings in the early stages included dilated intestinal loops, thickening of the intestinal wall, mesenteric fat edema, and air-fluid levels. Once the viability of the affected intestinal segment is compromised, a CT exam may evidence pneumatosis as a sign of bacterial proliferation and translocation in the intestinal wall, pneumoperitoneum due to perforation, and free fluid in the abdominal cavity. In cases with an unconfirmed diagnosis of COVID-19, examination of the pulmonary basis during abdominal CT exam can add consistent findings to establish the diagnosis.

Venous thrombosis affecting the superior mesenteric vein and or portal vein was encountered in $40.9 \%$ of reported cases of non-hospitalized COVID-19 patients, and in only one case in the hospitalized group (Table 5). One explanation may be the beneficial role of thrombotic prophylaxis in preventing venous thrombosis in COVID-19 patients, which is routinely administrated in hospitalized cases, but not reported in cases treated at home with COVID-19 pneumonia.

In ICU patients, CT exam showed in most cases permeable mesenteric vessels and diffuse intestinal ischemia affecting the large bowel alone (56\%) or in association with the small bowel (24\%), suggesting pathogenic mechanisms, direct viral infection, small vessel thrombosis, or "nonocclusive mesenteric ischemia" [16].

\subsection{Management and Outcomes}

The management of mesenteric ischemia includes gastrointestinal decompression, fluid resuscitation, hemodynamic support, anticoagulation, and broad antibiotics.

Once the thromboembolic event was diagnosed, heparin, 5000IU iv, or enoxaparin or LMWH in therapeutic doses was initiated, followed by long-term oral anticoagulation and/or anti-aggregating therapy. Favorable results were obtained in 7 out of 9 cases (77\%) of splanchnic veins thrombosis and in 2 of 7 cases $(28.5 \%)$ with superior mesenteric artery thrombosis. At discharge, anticoagulation therapy was continued either with LMWH, for a period up to 3 months $[33,36,41]$, either, long term warfarin, with INR control $[32,34,41]$ or apixaban $5 \mathrm{mg} /$ day, up to 6 months [26,47]. No readmissions were reported. 
Table 5. Comparative features in acute intestinal ischemia encountered in previously hospitalized and previously non-hospitalized COVID-19 patients.

\begin{tabular}{|c|c|c|c|}
\hline Parameter & $\begin{array}{c}\text { Hospitalized } \\
\text { (63) }\end{array}$ & $\begin{array}{c}\text { Non- } \\
\text { Hospitalized } \\
\text { (26) }\end{array}$ & $p *$ Value \\
\hline 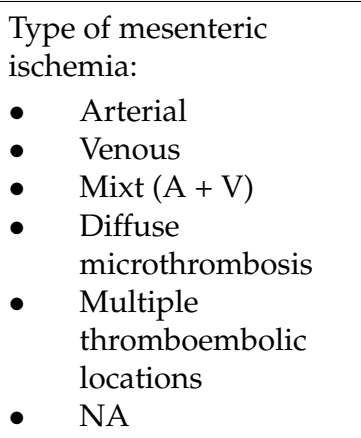 & $\begin{array}{l}5(14.7 \% *) \\
1(2.9 \%) \\
0 \\
30(88.2 \%) \\
2(5.8 \%) \\
29\end{array}$ & $\begin{array}{l}10(38.4 \%) \\
11(42.3 \%) \\
2(7.6 \%) \\
3(11.5 \%) \\
1(3.8 \%) \\
0\end{array}$ & $p<0.0001$ \\
\hline $\begin{array}{l}\text { Management: } \\
\text { - } \quad \text { Anticoagulation } \\
\text { therapy only } \\
\text { - } \quad \text { Endovascular } \\
\text { thrombectomy } \\
\text { - } \quad \text { Laparotomy with } \\
\text { ischemic bowel } \\
\text { resection } \\
\text { - None (fulminant } \\
\text { evolution) }\end{array}$ & $\begin{array}{l}0 \\
2(1+\text { surgery) }(3 \%) \\
60(95.4 \%) \\
2(3 \%)\end{array}$ & $\begin{array}{l}10(38.4 \%) \\
2 \text { (+surgery) } \\
15(57.6 \%) \\
1(3.8 \%)\end{array}$ & $p<0.0001$ \\
\hline $\begin{array}{l}\text { Location of the resected } \\
\text { segment: } \\
\text { - } \quad \text { Colon } \\
\text { - } \quad \text { Small bowel } \\
\text { - } \quad \text { Colon+small bowel } \\
\text { - } \quad \text { NA }\end{array}$ & $\begin{array}{l}35(56 \%) \\
10(16 \%) \\
15(24 \%) \\
6\end{array}$ & $\begin{array}{l}0 \\
12(80 \%) \\
3(20 \%) \\
0\end{array}$ & $p<0.0001$ \\
\hline $\begin{array}{l}\text { Outcomes: } \\
\text { - } \quad \text { Recovery } \\
\text { - } \quad \text { Death } \\
\text { - } \quad \text { NA }\end{array}$ & $\begin{array}{l}26(46.4 \%) \\
30(54.4 \%) \\
7\end{array}$ & $\begin{array}{l}17(79.3 \%) \\
5(21.7 \%) \\
3\end{array}$ & $p=0.013$ \\
\hline
\end{tabular}

Antibiotic classes should cover anaerobes including F. necrophorum and include a combination of beta-lactam and beta-lactamase inhibitor (e.g., piperacillin-tazobactam), metronidazole, ceftriaxone, clindamycin, and carbapenems [4].

In early diagnosis, during the first $12 \mathrm{~h}$ from the onset, vascular surgery may be tempted, avoiding the enteral resection $[25,53]$. Endovascular management is a minimally invasive approach, allowing quick restoration of blood flow in affected vessels using techniques such as aspiration, thrombectomy, thrombolysis, and angioplasty with or without stenting [40].

Laparotomy with resection of the necrotic bowel should be performed as quickly as possible to avoid perforation and septic shock. In cases in which intestinal viability cannot be established with certainty, a second look laparotomy was performed after 24-48 h [43] or the abdominal cavity was left open, using negative pressure systems such as ABTHERA [51], and successive segmentary enterectomy was performed.

Several authors described in acute bowel ischemia encountered in ICU patients with COVID-19, a distinct yellowish color, rather than the typical purple or black color of ischemic bowel, predominantly located at the antimesenteric side or circumferentially with affected areas well delineated from the adjacent healthy areas [18,19]. In these cases, patency of large mesenteric vessels was confirmed, and the histopathological reports 
showed endothelitis, inflammation, and microvascular thrombosis in the submucosa or transmural. Despite early surgery, the outcome is severe in these cases, with an overall mortality of $45-50 \%$ in reported studies and up to $100 \%$ in patients over 65 years of age according to Hwabejira et al. [19].

In COVID-19 patients non hospitalized at the onset of an acute ischemic event, with mild and moderate forms of the disease, the outcome was less severe, with recovery in $77 \%$ of cases.

We found that age over 60 years and the necessity of surgical treatment are statistically correlated with a poor outcome in the reviewed studies (Table 6). According to the type of mesenteric ischemia, the venous thrombosis was more likely to have a favorable outcome (recovery in $80 \%$ of cases), while vascular micro thombosis lead to death in $66 \%$ of cases.

Table 6. Risk factors for severe outcome.

\begin{tabular}{|c|c|c|}
\hline Parameters & Outcome: Death & $p$-Value \\
\hline $\begin{array}{ll}\text { Age } & \\
\text { - } & \text { Age }<60 \\
\text { - } & \text { Age }>60\end{array}$ & $\begin{array}{l}27.2 \% \\
60 \%\end{array}$ & $\begin{array}{l}0.0384 \text { * } \\
0.043 \text { ** }\end{array}$ \\
\hline $\begin{array}{l}\text { Surgery } \\
-\quad \text { No surgery } \\
\bullet \quad \text { surgery } \\
\end{array}$ & $\begin{array}{l}0 \% \\
60 \%\end{array}$ & $0.019 * *$ \\
\hline \begin{tabular}{ll}
\multicolumn{2}{l}{ Type of mesenteric ischemia } \\
- & Arterial \\
- & Venous \\
- & Micro thrombosis
\end{tabular} & $\begin{array}{l}47 \% \\
20 \% \\
66 \%\end{array}$ & 0.23 ** \\
\hline D dimers & Wide variation & $\begin{array}{l}0.085^{*} \\
0.394^{* *}\end{array}$ \\
\hline Leucocytes & $\begin{array}{l}\text { Wide variation } \\
(9650-37,000 / \mathrm{mmc})\end{array}$ & $\begin{array}{l}0.803 \\
0.385 \text { ** }\end{array}$ \\
\hline
\end{tabular}

* One-way ANOVA test; ${ }^{* *}$ Chi-squared test (SciStat ${ }^{\circledR}$ software, www.scistat.com (accessed on 25 November 2021)).

\section{Discussions}

Classically, acute mesenteric ischemia is a rare surgical emergency encountered in the elderly with cardiovascular or portal-associated pathology, such as arterial hypertension, atrial fibrillation, atherosclerosis, heart failure, valve disease, and portal hypertension. However, in the current context of the COVID-19 pandemic, mesenteric ischemia should be suspected in any patient presenting in an emergency with acute abdominal pain, regardless of age and associated diseases.

Several biomarkers were investigated for the potential diagnostic and prognostic value in acute mesenteric ischemia. Serum lactate is a non-specific biomarker of tissue hypoperfusion and undergoes significant elevation only after advanced mesenteric damage. Several clinical trials found a value higher than $2 \mathrm{mmol} / \mathrm{L}$ was significantly associated with increased mortality in non-COVID-patients. However, its diagnostic value is still a subject of debate. There are two detectable isomers, L-lactate, which is a nonspecific biomarker of anaerobic metabolism, and hypoxia and D-lactate, which is produced by the activity of intestinal bacteria. Higher D-lactate levels could be more specific for mesenteric ischemia due to increased bacterial proliferation at the level of the ischemic bowel, but the results obtained in different studies are mostly inconsistent [53,54].

Several clinical studies found that LDH is a useful biomarker for acute mesenteric ischemia, [55,56]. However, interpretation of the results may be difficult in COVID-19 patients, as both lactate and LDH were also found to be independent risk factors of severe forms of COVID-19 [57,58].

The diagnosis of an ischemic bowel should be one of the top differentials in critically ill patients with acute onset of abdominal pain and distension [50,59]. If diagnosed early, the 
intestinal ischemia is potentially reversible and can be treated conservatively. Heparin has an anticoagulant, anti-inflammatory, endothelial protective role in COVID-19, which can improve microcirculation and decrease possible ischemic events [25]. The appropriate dose, however, is still a subject of debate with some authors recommending the prophylactic, others the intermediate or therapeutic daily amount $[25,60]$.

We found that surgery is associated with a severe outcome in the reviewed studies. Mucosal ischemia may induce massive viremia from bowel epithelium causing vasoplegic shock after surgery [25]. Moreover, many studies reported poor outcomes in COVID-19 patients that underwent abdominal surgery [61,62].

\subsection{Pathogenic Pathways of Mesenteric Ischemia in COVID-19 Patients}

The intestinal manifestations encountered in SARS-CoV-2 infection are represented by inflammatory changes (gastroenteritis, colitis), occlusions, ileus, invaginations, and ischemic manifestations. Severe inflammation in the intestine can cause damage to the submucosal vessels, resulting in hypercoagulability in the intestine. Cases of acute cholecystitis, splenic infarction, or acute pancreatitis have also been reported in patients infected with SARS-CoV-2, with microvascular lesions as a pathophysiological mechanism [63].

In the study of O'Shea et al., on 146 COVID-19 hospitalized patients that underwent CT-scan, vascular thrombosis was identified in $26 \%$ of cases, the most frequent location being in lungs [20]. Gastrointestinal ischemic lesions were identified in 4 cases, in multiple locations (pulmonary, hepatic, cerebellar parenchymal infarction) in 3 patients. The authors raised awareness about the possibility of underestimation of the incidence of thrombotic events in COVID-19 patients [20].

Several pathophysiological mechanisms have been considered, and they can be grouped into occlusive and non-occlusive causes [64]. The site of the ischemic process, embolism or thrombosis, may be in the micro vascularization, veins, or mesenteric arteries.

Acute arterial obstruction of the small intestinal vessels and mesenteric ischemia may appear due to hypercoagulability associated with SARS-CoV-2 infection, mucosal ischemia, viral dissemination, and endothelial cell invasion vis ACE-2 receptors [65,66]. Viral binding to ACE2Receptors leads to significant changes in fluid-coagulation balance: reduction in Ang 2 degradation leads to increased Il6 levels, and the onset of storm cytokines, such as IL-2, IL-7, IL-10, granulocyte colony-stimulating factor, IgG -induced protein 10, monocyte chemoattractant protein-1, macrophage inflammatory protein 1-alpha, and tumor necrosis factor $\alpha$ [67], but also in the expression of the tissue inhibitor of plasminogen -1 , and a tissue factor, and subsequently triggering the coagulation system through binding to the clotting factor VIIa [68]. Acute embolism in small vessels may be caused by the direct viral invasion, via ACE-2 Receptors, resulting in endothelitis and inflammation, recruiting immune cells, and expressing high levels of pro-inflammatory cytokines, such as Il-6 and TNF-alfa, with consequently apoptosis of the endothelial cells [69].

Capillary viscometry showed hyperviscosity in critically ill COVID-19 patients [70,71] Platelet activation, platelet-monocyte aggregation formation, and Neutrophil external traps (NETs) released from activated neutrophils, constitute a mixture of nucleic DNA, histones, and nucleosomes [59,72] were documented in severe COVID-19 patients by several studies $[70,71,73]$.

Plotz et al. found a thrombotic vasculopathy with histological evidence for lectin pathway complement activation mirroring viral protein deposition in a patient with COVID19 and SLE, suggesting this might be a potential mechanism in SARS-CoV-2 associated thrombotic disorders [47].

Numerous alterations in fluid-coagulation balance have been reported in patients hospitalized for COVID-19 pneumonia. Increases in fibrinogen, D-dimers, but also coagulation factors V and VIII. The mechanisms of coagulation disorders in COVID-19 are not yet fully elucidated. In a clinical study by Stefely et al. [68] in a group of 102 patients with severe disease, an increase in factor $\mathrm{V}>200$ IU was identified in $48 \%$ of cases, the levels determined being statistically significantly higher than in non-COVID mechanically 
ventilated or unventilated patients hospitalized in intensive care. This showed that the increased activity of Factor $V$ cannot be attributed to disease severity or mechanical ventilation. Additionally, an increase in factor $X$ activity was shown, but not correlated with an increase in factor $\mathrm{V}$ activity, but with an increase in acute phase reactants, suggesting distinct pathophysiological mechanisms [74].

Giuffre et al. suggest that fecal calcoprotein (FC) may be a biomarker for the severity of gastrointestinal complications, by both ischemic and inflammatory mechanisms [75]. They found particularly elevated levels of FC to be well correlated with D-dimers levels in patients with bowel perforations, and hypothesized that the mechanism may be related to a thrombosis localized to the gut and that FC increase is related to virus-related inflammation and thrombosis-induced ischemia, as shown by gross pathology [76].

Non-occlusive mesenteric ischemia in patients hospitalized in intensive care units for SARS-CoV-2 pneumonia requiring vasopressor medication may be caused vasospastic constriction $[19,64,65]$. Thrombosis of the mesenteric vessels could be favored by hypercoagulability, relative dehydration, and side effects of corticosteroids.

\subsection{Question Still to Be Answered}

Current recommendations for in-hospital patients with COVID-19 requiring anticoagulation suggest LMWH as first-line treatment has advantages, with higher stability compared to heparin during cytokine storms, and a reduced risk of interaction with antiviral therapy compared to oral anticoagulant medication [77]. Choosing the adequate doses of LMWH in specific cases-prophylactic, intermediate, or therapeutic-is still in debate. Thromboprophylaxis is highly recommended in the absence of contraindications, due to the increased risk of venous thrombosis and arterial thromboembolism associated with SARS-CoV-2 infection, with dose adjustment based on weight and associated risk factors. Besides the anticoagulant role, some authors also reported an anti-inflammatory role of heparin in severe COVID-19 infection [66,78,79]. Heparin is known to decrease inflammation by inhibiting neutrophil activity, expression of inflammatory mediators, and the proliferation of vascular smooth muscle cells [78]. Thromboprophylaxis with enoxaparin could be also recommended to ambulatory patients with mild to moderate forms of COVID-19 if the results of prospective studies show statistically relevant benefits [80].

In addition to anticoagulants, other therapies, such as anti-complement and interleukin (IL)-1 receptor antagonists, need to be explored, and other new agents should be discovered as they emerge from our better understanding of the pathogenetic mechanisms [81]. Several studies showed the important role of Il-1 in endothelial dysfunction, inflammation, and thrombi formation in COVID-19 patients by stimulating the production of Thromboxane A2 (TxA2) and thromboxane B2 (TxB2). These findings may justify the recommendation for an IL-1 receptor antagonist (IL-1Ra) which can prevent hemodynamic changes, septic shock, organ inflammation, and vascular thrombosis in severe forms of COVID-19 patients [80-82].

\section{Conclusions}

Understanding the pathological pathways and risk factors could help adjust the thromboprophylaxis and fluid management in COVID-19 patients. The superior mesenteric vein thrombosis is the most frequent cause of acute intestinal ischemia in COVID-19 nonhospitalized patients that are not under anticoagulant medication, while non-occlusive mesenteric ischemia and microvascular thrombosis are most frequent in severe cases, hospitalized in intensive care units.

COVID-19 patients should be carefully monitored for acute onset of abdominal symptoms. High-intensity pain and abdominal distension, associated with leukocytosis, raised inflammatory biomarkers and elevated D-dimers and are highly suggestive for mesenteric ischemia. The contrast-enhanced CT exam, repeated, if necessary, offers valuable information regarding the location and extent of the acute ischemic event. Early diagnosis and treatment are essential for survival. 
Supplementary Materials: The following supporting information can be downloaded at: https: / / www.mdpi.com/article/10.3390/jcm11010200/s1, File S1: The PRISMA 2020 statement.

Author Contributions: Conceptualization, D.S., L.C.T. and A.M.D.; methodology, A.P.S., C.T. (Corneliu Tudor); software, G.V.; validation, A.I.S., M.S.T., D.S. and L.D.; formal analysis, A.C.C., C.T. (Ciprian Tanasescu); investigation, G.A.G.; data curation, D.O.C.; writing-original draft preparation, L.C.T., A.M.D., G.V., D.O.C., G.A.G., C.T. (Corneliu Tudor); writing-review and editing, L.D., C.T. (Ciprian Tanasescu), A.C.C., D.S., A.P.S., A.I.S., M.S.T.; visualization, G.V. and L.C.T.; supervision, D.S., A.M.D. and D.S. have conducted the screening and selection of studies included in the review All authors have read and agreed to the published version of the manuscript.

Funding: This research received no external funding.

Conflicts of Interest: The authors declare no conflict of interest.

\section{References}

1. Bala, M.; Kashuk, J.; Moore, E.E.; Kluger, Y.; Biffl, W.; Gomes, C.A.; Ben-Ishay, O.; Rubinstein, C.; Balogh, Z.J.; Civil, I.; et al. Acute mesenteric ischemia: Guidelines of the World Society of Emergency Surgery. World J. Emerg. Surg. 2017, 12, 38. [CrossRef]

2. Dumic, I.; Martin, S.; Salfiti, N.; Watson, R.; Alempijevic, T. Deep Venous Thrombosis and Bilateral Pulmonary Embolism Revealing Silent Celiac Disease: Case Report and Review of the Literature. Case Rep. Gastrointest. Med. 2017, 2017, 5236918. [CrossRef] [PubMed]

3. Akhrass, F.A.; Abdallah, L.; Berger, S.; Sartawi, R. Gastrointestinal variant of Lemierre's syndrome complicating ruptured appendicitis. IDCases 2015, 2, 72-76. [CrossRef]

4. Radovanovic, N.; Dumic, I.; Veselinovic, M.; Burger, S.; Milovanovic, T.; Nordstrom, C.W.; Niendorf, E.; Ramanan, P. Fusobacterium necrophorum subsp. necrophorum Liver Abscess with Pylephlebitis: An Abdominal Variant of Lemierre's Syndrome. Case Rep. Infect. Dis. 2020, 2020, 9237267. [CrossRef]

5. Sogaard, K.K.; Astrup, L.B.; Vilstrup, H.; Gronbaek, H. Portal vein thrombosis; risk factors, clinical presentation and treatment. BMC Gastroenterol. 2007, 7, 34. [CrossRef] [PubMed]

6. Moradi, H.; Mouzannar, S.; Miratashi Yazdi, S.A. Post COVID-19 splenic infarction with limb ischemia: A case report. Ann. Med. Surg. 2021, 71, 102935. [CrossRef] [PubMed]

7. $\quad$ Elmunzer, B.J.; Spitzer, R.L.; Foster, L.D.; Merchant, A.A.; Howard, E.F.; Patel, V.A.; West, M.K.; Qayed, E.; Nustas, R.; Zakaria, A.; et al. North American Alliance for the Study of Digestive Manifestations of COVID-19. Digestive Manifestations in Patients Hospitalized With Coronavirus Disease 2019. Clin. Gastroenterol. Hepatol. 2021, 19, 1355-1365.e4. [CrossRef]

8. Guan, W.J.; Ni, Z.Y.; Hu, Y. Clinical characteristics of coronavirus disease 2019 in China. N. Engl. J. Med. 2020, 382, 1708-1720. [CrossRef]

9. Estevez-Cerda, S.C.; Saldaña-Rodríguez, J.A.; Alam-Gidi, A.G.; Riojas-Garza, A.; Rodarte-Shade, M.; Velazco-de la Garza, J.; Leyva-Alvizo, A.; Gonzalez-Ruvalcaba, R.; Martinez-Resendez, M.F.; Ortiz de Elguea-Lizarraga, J.I. Severe bowel complications in SARS-CoV-2 patients receiving protocolized care. Rev. Gastroenterol. Mex. Engl. Ed. 2021, 86, 378-386. [CrossRef]

10. Redd, W.D.; Zhou, J.C.; Hathorn, K.E. Prevalence and characteristics of gastrointestinal symptoms in patients with SARS-CoV-2 infection in the United States: A multicenter cohort study. Gastroenterology 2020, 159, 765-767.e2. [CrossRef]

11. Hajifathalian, K.; Krisko, T.; Mehta, A. Gastrointestinal and hepatic manifestations of 2019 novel coronavirus disease in a large cohort of infected patients from New York: Clinical implications. Gastroenterology 2020, 159, 1137-1140.e2. [CrossRef]

12. Kotfis, K.; Skonieczna-Żydecka, K. COVID-19: Gastrointestinal symptoms and potential sources of SARS-CoV-2 transmission. Anaesthesiol. Intensive Ther. 2020, 52, 171-172. [CrossRef]

13. Xiao, F.; Tang, M.; Zheng, X. Evidence for gastrointestinal infection of SARS-CoV-2. Gastroenterology 2020, 158, 1831-1833. [CrossRef] [PubMed]

14. Xu, Y.; Li, X.; Zhu, B.; Liang, H.; Fang, C.; Gong, Y.; Guo, Q.; Sun, X.; Zhao, D.; Shen, J.; et al. Characteristics of pediatric SARS-CoV-2 infection and potential evidence for persistent fecal viral shedding. Nat. Med. 2020, 26, 502-505. [CrossRef] [PubMed]

15. Ludewig, S.; Jarbouh, R.; Ardelt, M.; Mothes, H.; Rauchfuß, F.; Fahrner, R.; Zanow, J.; Settmacher, U. Bowel Ischemia in ICU Patients: Diagnostic Value of I-FABP Depends on the Interval to the Triggering Event. Gastroenterol. Res. Pract. 2017, 2795176. [CrossRef]

16. Kaafarani, H.; El Moheb, M.; Hwabejire, J.O.; Naar, L.; Christensen, M.A.; Breen, K.; Gaitanidis, A.; Alser, O.; Mashbari, H.; Bankhead-Kendall, B.; et al. Gastrointestinal Complications in Critically Ill Patients With COVID-19. Ann. Surg. 2020, 272, e61-e62. [CrossRef]

17. Kraft, M.; Pellino, G.; Jofra, M.; Sorribas, M.; Solís-Peña, A.; Biondo, S.; Espín-Basany, E. Incidence, features, outcome and impact on health system of de-novo abdominal surgical diseases in patients admitted with COVID-19. Surg. J. R. Coll. Surg. Edinb. Irel. 2021, 19, e53-e58. [CrossRef]

18. Yang, C.; Hakenberg, P.; Weiß, C.; Herrle, F.; Rahbari, N.; Reißfelder, C.; Hardt, J. Colon ischemia in patients with severe COVID-19: A single-center retrospective cohort study of 20 patients. Int. J. Colorectal Dis. 2021, 36, 2769-2773. [CrossRef] 
19. Hwabejire, J.O.; Kaafarani, H.M.; Mashbari, H.; Misdraji, J.; Fagenholz, P.J.; Gartland, R.M.; Abraczinskas, D.R.; Mehta, R.S.; Paranjape, C.N.; Eng, G.; et al. Bowel Ischemia in COVID-19 Infection: One-Year Surgical Experience. Am. Surg. 2021, 87, 1893-1900. [CrossRef] [PubMed]

20. O'shea, A.; Parakh, A.; Hedgire, S.; Lee, S.I. Multisystem assessment of the imaging manifestations of coagulopathy in hospitalized patients with coronavirus. Am. J. Roentgenol. 2021, 216, 1088-1098. [CrossRef] [PubMed]

21. Qayed, E.; Deshpande, A.R.; Elmunzer, B.J.; North American Alliance for the Study of Digestive Manifestations of COVID-19. Low Incidence of Severe Gastrointestinal Complications in COVID-19 Patients Admitted to the Intensive Care Unit: A Large, Multicenter Study. Gastroenterology 2021, 160, 1403-1405. [CrossRef] [PubMed]

22. Azouz, E.; Yang, S.; Monnier-Cholley, L.; Arrivé, L. Systemic arterial thrombosis and acute mesenteric ischemia in a patient with COVID-19. Intensive Care Med. 2020, 46, 1464-1465. [CrossRef] [PubMed]

23. Al Mahruqi, G.; Stephen, E.; Abdelhedy, I.; Al Wahaibi, K. Our early experience with mesenteric ischemia in COVID-19 positive patients. Ann. Vasc. Surg. 2021, 73, 129-132. [CrossRef] [PubMed]

24. Ucpinar, B.A.; Sahin, C. Superior Mesenteric Artery Thrombosis in a Patient with COVID-19: A Unique Presentation. J. Coll Physicians Surg. Pak. 2020, 30, 112-114. [CrossRef]

25. Karna, S.T.; Panda, R.; Maurya, A.P.; Kumari, S. Superior Mesenteric Artery Thrombosis in COVID-19 Pneumonia: An Underestimated Diagnosis-First Case Report in Asia. Indian J. Surg. 2020, 82, 1235-1237. [CrossRef]

26. Abeysekera, K.W.; Karteszi, H.; Clark, A.; Gordon, F.H. Spontaneous portomesenteric thrombosis in a non-cirrhotic patient with SARS-CoV-2 infection. BMJ Case Rep. 2020, 13, e238906. [CrossRef]

27. Rodriguez-Nakamura, R.M.; Gonzalez-Calatayud, M.; Martinez Martinez, A.R. Acute mesenteric thrombosis in two patients with COVID-19. Two cases report and literature review. Int. J. Surg. Case Rep. 2020, 76, 409-414. [CrossRef]

28. Dinoto, E.; Ferlito, F.; La Marca, M.A.; Mirabella, D.; Bajardi, G.; Pecoraro, F. Staged acute mesenteric and peripheral ischemia treatment in COVID-19 patient: Case report. Int. J. Surg. Case Rep. 2021, 84, 106105. [CrossRef]

29. Kiwango, F.; Mremi, A.; Masenga, A.; Akrabi, H. Intestinal ischemia in a COVID-19 patient: Case report from Northern Tanzania. J. Surg. Case Rep. 2021, 2021, rjaa537. [CrossRef]

30. Sevella, P.; Rallabhandi, S.; Jahagirdar, V.; Kankanala, S.R.; Ginnaram, A.R.; Rama, K. Acute Mesenteric Ischemia as an Early Complication of COVID-19. Cureus 2021, 13, e18082. [CrossRef]

31. Nasseh, S.; Trabelsi, M.M.; Oueslati, A.; Haloui, N.; Jerraya, H.; Nouira, R. COVID-19 and gastrointestinal symptoms: A case report of a Mesenteric Large vessel obstruction. Clin. Case Rep. 2021, 9, e04235. [CrossRef] [PubMed]

32. Alemán, W.; Cevallos, L.C. Subacute mesenteric venous thrombosis secondary to COVID-19: A late thrombotic complication in a nonsevere patient. Radiol. Case Rep. 2021, 16, 899-902. [CrossRef] [PubMed]

33. Jeilani, M.; Hill, R.; Riad, M.; Abdulaal, Y. Superior mesenteric vein and portal vein thrombosis in a patient with COVID-19: A rare case. BMJ Case Rep. 2021, 14, e244049. [CrossRef]

34. Randhawa, J.; Kaur, J.; Randhawa, H.S.; Kaur, S.; Singh, H. Thrombosis of the Portal Vein and Superior Mesenteric Vein in a Patient With Subclinical COVID-19 Infection. Cureus 2021, 13, e14366. [CrossRef] [PubMed]

35. Cheung, S.; Quiwa, J.C.; Pillai, A.; Onwu, C.; Tharayil, Z.J.; Gupta, R. Superior Mesenteric Artery Thrombosis and Acute Intestinal Ischemia as a Consequence of COVID-19 Infection. Am. J. Case Rep. 2020, 21, e925753. [CrossRef]

36. Beccara, L.A.; Pacioni, C.; Ponton, S.; Francavilla, S.; Cuzzoli, A. Arterial Mesenteric Thrombosis as a Complication of SARS-CoV-2 Infection. Eur. J. Case Rep. Intern. Med. 2020, 7, 001690. [CrossRef] [PubMed]

37. Vulliamy, P.; Jacob, S.; Davenport, R.A. Acute aorto-iliac and mesenteric arterial thromboses as presenting features of COVID-19. Br. J. Haematol. 2020, 189, 1053-1054. [CrossRef]

38. De Barry, O.; Mekki, A.; Diffre, C.; Seror, M.; El Hajjam, M.; Carlier, R.Y. Arterial and venous abdominal thrombosis in a 79-year-old woman with COVID-19 pneumonia. Radiol. Case Rep. 2020, 15, 1054-1057. [CrossRef]

39. Romero, M.D.C.V.; Cárdenas, A.M.; Fuentes, A.B.; Barragán, A.A.S.; Gómez, D.B.S.; Jiménez, M.T. Acute mesenteric arterial thrombosis in severe SARS-Co-2 patient: A case report and literature review. Int. J. Surg. Case Rep. 2021, 86, 106307. [CrossRef]

40. Posada-Arango, A.M.; García-Madrigal, J.; Echeverri-Isaza, S.; Alberto-Castrillón, G.; Martínez, D.; Gómez, A.C.; Pinto, J.A.; Pinillos, L. Thrombosis in abdominal vessels associated with COVID-19 Infection: A report of three cases. Radiol. Case Rep. 2021, 16, 3044-3050. [CrossRef]

41. Pang, J.H.Q.; Tang, J.H.; Eugene-Fan, B. A peculiar case of small bowel stricture in a coronavirus disease 2019 patient with congenital adhesion band and superior mesenteric vein thrombosis. Ann. Vasc. Surg. 2021, 70, 286-289. [CrossRef]

42. Lari, E.; Lari, A.; AlQinai, S. Severe ischemic complications in COVID-19-a case series. Int. J. Surg. Case Rep. 2020, 75, 131-135. [CrossRef] [PubMed]

43. Carmo Filho, A.; Cunha, B.D.S. Inferior mesenteric vein thrombosis and COVID-19. Rev. Soc. Bras. Med. Trop. 2020, 53, e20200412. [CrossRef]

44. Hanif, M.; Ahmad, Z.; Khan, A.W.; Naz, S.; Sundas, F. COVID-19-Induced Mesenteric Thrombosis. Cureus 2021, 13, e12953. [CrossRef]

45. Amaravathi, U.; Balamurugan, N.; Muthu Pillai, V.; Ayyan, S.M. Superior Mesenteric Arterial and Venous Thrombosis in COVID-19. J. Emerg. Med. 2021, 60, e103-e107. [CrossRef] [PubMed]

46. Goodfellow, M.; Courtney, M.; Upadhyay, Y.; Marsh, R.; Mahawar, K. Mesenteric Venous Thrombosis Due to Coronavirus in a Post Roux-en-Y Gastric Bypass Patient: A Case Report. Obes. Surg. 2021, 31, 2308-2310. [CrossRef] [PubMed] 
47. Plotz, B.; Castillo, R.; Melamed, J.; Magro, C.; Rosenthal, P.; Belmont, H.M. Focal small bowel thrombotic microvascular injury in COVID-19 mediated by the lectin complement pathway masquerading as lupus enteritis. Rheumatology 2021, 60, e61-e63. [CrossRef]

48. Chiu, C.Y.; Sarwal, A.; Mon, A.M.; Tan, Y.E.; Shah, V. Gastrointestinal: COVID-19 related ischemic bowel disease. J. Gastroenterol. Hepatol. 2021, 36, 850. [CrossRef] [PubMed]

49. Farina, D.; Rondi, P.; Botturi, E.; Renzulli, M.; Borghesi, A.; Guelfi, D.; Ravanelli, M. Gastrointestinal: Bowel ischemia in a suspected coronavirus disease (COVID-19) patient. J. Gastroenterol. Hepatol. 2021, 36, 41. [CrossRef]

50. Singh, B.; Mechineni, A.; Kaur, P.; Ajdir, N.; Maroules, M.; Shamoon, F.; Bikkina, M. Acute Intestinal Ischemia in a Patient with COVID-19 Infection. Korean J. Gastroenterol. 2020, 76, 164-166. [CrossRef]

51. Nakatsutsumi, K.; Endo, A.; Okuzawa, H.; Onishi, I.; Koyanagi, A.; Nagaoka, E.; Morishita, K.; Aiboshi, J.; Otomo, Y. Colon perforation as a complication of COVID-19: A case report. Surg. Case Rep. 2021, 7, 175. [CrossRef]

52. Carubbi, F.; Salvati, L.; Alunno, A.; Maggi, F.; Borghi, E.; Mariani, R.; Mai, F.; Paoloni, M.; Ferri, C.; Desideri, G.; et al. Ferritin is associated with the severity of lung involvement but not with worse prognosis in patients with COVID-19: Data from two Italian COVID-19 units. Sci. Rep. 2021, 11, 4863. [CrossRef]

53. Isfordink, C.J.; Dekker, D.; Monkelbaan, J.F. Clinical value of serum lactate measurement in diagnosing acute mesenteric ischaemia. Neth. J. Med. 2018, 76, 60-64. [PubMed]

54. Montagnana, M.; Danese, E.; Lippi, G. Biochemical markers of acute intestinal ischemia: Possibilities and limitations. Ann. Transl. Med. 2018, 6, 341. [CrossRef]

55. Matsumoto, S.; Sekine, K.; Funaoka, H.; Yamazaki MShimizu, M.; Hayashida, K.; Kitano, M. Diagnostic performance of plasma biomarkers in patients with acute intestinal ischaemia. Br. J. Surg. 2014, 101, 232-238. [CrossRef] [PubMed]

56. Soni, N.; Bhutra, S.; Vidyarthi, S.H.; Sharma, V. Role of serum lactic dehydrogenase, glutamic oxaloacetic transaminase, creatine phosphokinase, alkaline phospatase, serum phosphorus in the cases of bowel Ischaemia in acute abdomen. Int. Surg. J. 2017, 4, 1997-2001. [CrossRef]

57. Han, Y.; Zhang, H.; Mu, S.; Wei, W.; Jin, C.; Tong, C.; Song, Z.; Zha, Y.; Xue, Y.; Gu, G. Lactate dehydrogenase, an independent risk factor of severe COVID-19 patients: A retrospective and observational study. Aging 2020, 12, 11245-11258. [CrossRef]

58. Carpenè, G.; Onorato, D.; Nocini, R.; Fortunato, G.; Rizk, J.G.; Henry, B.M.; Lippi, G. Blood lactate concentration in COVID-19: A systematic literature review. Clin. Chem. Lab. Med. 2021. advance online publication. [CrossRef]

59. Singh, B.; Kaur, P.; Maroules, M. Splanchnic vein thrombosis in COVID-19: A review of literature. Dig. Liver Dis. 2020, 52, 1407-1409. [CrossRef]

60. Jagielski, M.; Piątkowski, J.; Jackowski, M. Challenges encountered during the treatment of acute mesenteric ischemia. Gastroenterol. Res. Pract. 2020, 5316849. [CrossRef] [PubMed]

61. Rasslan, R.; Dos Santos, J.P.; Menegozzo, C.; Pezzano, A.; Lunardeli, H.S.; Dos Santos Miranda, J.; Utiyama, E.M.; Damous, S. Outcomes after emergency abdominal surgery in COVID-19 patients at a referral center in Brazil. Updates Surg. 2021, 73, 763-768. [CrossRef]

62. Lei, S.; Jiang, F.; Su, W.; Chen, C.; Chen, J.; Mei, W.; Zhan, L.Y.; Jia, Y.; Zhang, L.; Liu, D.; et al. Clinical characteristics and outcomes of patients undergoing surgeries during the incubation period of COVID-19 infection. EClinicalMedicine 2020, 21, 100331. [CrossRef]

63. Serban, D.; Socea, B.; Badiu, C.D.; Tudor, C.; Balasescu, S.A.; Dumitrescu, D.; Trotea, A.M.; Spataru, R.I.; Vancea, G.; Dascalu, A.M.; et al. Acute surgical abdomen during the COVID 19 pandemic: Clinical and therapeutic challenges. Exp. Ther. Med. 2021, 21, 519. [CrossRef] [PubMed]

64. Patel, S.; Parikh, C.; Verma, D.; Sundararajan, R.; Agrawal, U.; Bheemisetty, N.; Akku, R.; Sánchez-Velazco, D.; Waleed, M.S. Bowel ischaemia in COVID-19: A systematic review. Int. J. Clin. Pract. 2021, 75, e14930. [CrossRef] [PubMed]

65. Yantiss, R.K.; Qin, L.; He, B.; Crawford, C.V.; Seshan, S.; Patel, S.; Wahid, N.; Jessurun, J. Intestinal Abnormalities in Patients With SARS-CoV-2 Infection: Histopathologic Changes Reflect Mechanisms of Disease. Am. J. Surg. Pathol. 2021, 46, 89-96. [CrossRef] [PubMed]

66. McGonagle, D.; Bridgewood, C.; Ramanan, A.V.; Meaney, J.F.M.; Watad, A. COVID-19 vasculitis and novel vasculitis mimics. Lancet Rheumatol. 2021, 3, e224-e233. [CrossRef]

67. Huang, C.; Wang, Y.; Li, X. Clinical features of patients infected with 2019 novel coronavirus in Wuhan. China Lancet 2020, 395, 497-506. [CrossRef]

68. Avila, J.; Long, B.; Holladay, D.; Gottlieb, M. Thrombotic complications of COVID-19. Am. J. Emerg. Med. 2021, 39, 213-218. [CrossRef]

69. Varga, Z.; Flammer, A.J.; Steiger, P.; Haberecker, M.; Andermatt, R.; Zinkernagel, A.S.; Mehra, M.R.; Schuepbach, R.A.; Ruschitzka, F.; Moch, H. Endothelial cell infection and endotheliitis in COVID-19. Lancet 2020, 395, 1417-1418. [CrossRef]

70. Maier, C.L.; Truong, A.D.; Auld, S.C.; Polly, D.M.; Tanksley, C.L.; Duncan, A. COVID-19-associated hyperviscosity: A link between inflammation and thrombophilia? Lancet 2020, 395, 1758-1759. [CrossRef]

71. Miyara, S.J.; Becker, L.B.; Guevara, S.; Kirsch, C.; Metz, C.N.; Shoaib, M.; Grodstein, E.; Nair, V.V.; Jandovitz, N.; McCannMolmenti, A.; et al. Pneumatosis Intestinalis in the Setting of COVID-19: A Single Center Case Series From New York. Front. Med. 2021, 8, 638075. [CrossRef] [PubMed] 
72. Panigada, M.; Bottino, N.; Tagliabue, P.; Grasselli, G.; Novembrino, C.; Chantarangkul, V.; Pesenti, A.; Peyvandi, F.; Tripodi, A. Hypercoagulability of COVID-19 patients in intensive care unit: A report of thromboelastography findings and other parameters of hemostasis. J. Thromb. Haemost. 2020, 18, 1738-1742. [CrossRef]

73. Hottz, E.D.; Azevedo-Quintanilha, I.G.; Palhinha, L.; Teixeira, L.; Barreto, E.A.; Pão, C.R.; Righy, C.; Franco, S.; Souza, T.M.; Kurtz, P.; et al. Platelet activation and platelet-monocyte aggregate formation trigger tissue factor expression in patients with severe COVID-19. Blood J. Am. Soc. Hematol. 2020, 136, 1330-1341. [CrossRef]

74. Stefely, J.A.; Christensen, B.B.; Gogakos, T.; Cone Sullivan, J.K.; Montgomery, G.G.; Barranco, J.P.; Van Cott, E.M. Marked factor $\mathrm{V}$ activity elevation in severe COVID-19 is associated with venous thromboembolism. Am. J. Hematol. 2020, 95, 1522-1530. [CrossRef]

75. Giuffrè, M.; Di Bella, S.; Sambataro, G.; Zerbato, V.; Cavallaro, M.; Occhipinti, A.A.; Palermo, A.; Crescenti, A.; Monica, F.; Luzzati, R.; et al. COVID-19-Induced Thrombosis in Patients without Gastrointestinal Symptoms and Elevated Fecal Calprotectin: Hypothesis Regarding Mechanism of Intestinal Damage Associated with COVID-19. Trop. Med. Infect. Dis. 2020, 5, 147. [CrossRef] [PubMed]

76. Giuffrè, M.; Vetrugno, L.; Di Bella, S.; Moretti, R.; Berretti, D.; Crocè, L.S. Calprotectin and SARS-CoV-2: A Brief-Report of the Current Literature. Healthcare 2021, 9, 956. [CrossRef] [PubMed]

77. Buso, G.; Becchetti, C.; Berzigotti, A. Acute splanchnic vein thrombosis in patients with COVID-19: A systematic review. Dig. Liver Dis. 2021, 53, 937-949. [CrossRef]

78. Thachil, J. The versatile heparin in COVID-19. J. Thromb. Haemost. 2020, 18, 1020-1022. [CrossRef]

79. Poterucha, T.J.; Libby, P.; Goldhaber, S.Z. More than an anticoagulant: Do heparins have direct anti-inflammatory effects? Thromb. Haemost. 2017, 117, 437-444. [CrossRef]

80. Wang, M.K.; Yue, H.Y.; Cai, J.; Zhai, Y.J.; Peng, J.H.; Hui, J.F.; Hou, D.Y.; Li, W.P.; Yang, J.S. COVID-19 and the digestive system: A comprehensive review. World J. Clin. Cases 2021, 9, 3796-3813. [CrossRef]

81. Manolis, A.S.; Manolis, T.A.; Manolis, A.A.; Papatheou, D.; Melita, H. COVID-19 Infection: Viral Macro- and Micro-Vascular Coagulopathy and Thromboembolism/Prophylactic and Therapeutic Management. J. Cardiovasc. Pharmacol. Ther. 2021, $26,12-24$. [CrossRef] [PubMed]

82. Conti, P.; Caraffa, A.; Gallenga, C.E.; Ross, R.; Kritas, S.K.; Frydas, I.; Younes, A.; Di Emidio, P.; Ronconi, G.; Toniato, E. IL-1 induces throboxane-A2 (TxA2) in COVID-19 causing inflammation and micro-thrombi: Inhibitory effect of the IL-1 receptor antagonist (IL-1Ra). J. Biol. Regul. Homeost. Agents 2020, 34, 1623-1627. [CrossRef] [PubMed] 